\title{
SARS-CoV-2 spike D614G change enhances replication and transmission
}

https://doi.org/10.1038/s41586-021-03361-1

Received: 15 October 2020

Accepted: 16 February 2021

Published online: 26 February 2021

Check for updates

\author{
Bin Zhou ${ }^{1,12}$, Tran Thi Nhu Thao ${ }^{2,3,4,12}$, Donata Hoffmann ${ }^{5,12}$, Adriano Taddeo ${ }^{2,3,12}$, Nadine Ebert ${ }^{2,3}$, \\ Fabien Labroussaa ${ }^{3,6}$, Anne Pohlmann ${ }^{5}$, Jacqueline King ${ }^{5}$, Silvio Steiner ${ }^{2,3,4}$, Jenna N. Kelly ${ }^{2,3}$, \\ Jasmine Portmann ${ }^{2,3}$, Nico Joel Halwe ${ }^{5}$, Lorenz Ulrich ${ }^{5}$, Bettina Salome Trüeb ${ }^{3,6}$, Xiaoyu Fan', \\ Bernd Hoffmann ${ }^{5}$, Li Wang', Lisa Thomann ${ }^{2,3}$, Xudong Lin ${ }^{7}$, Hanspeter Stalder ${ }^{2,3}$, Berta Pozzi ${ }^{8}$, \\ Simone de Brot ${ }^{9}$, Nannan Jiang ${ }^{10}$, Dan Cui ${ }^{7}$, Jaber Hossain', Malania M. Wilson', \\ Matthew W. Keller', Thomas J. Stark', John R. Barnes', Ronald Dijkman ${ }^{2,3,11}$, Joerg Jores ${ }^{3,6}$, \\ Charaf Benarafa ${ }^{2,3,13 凶}$, David E. Wentworth ${ }^{1,13 凶}$, Volker Thiel ${ }^{2,3,13} \bowtie$ \& Martin Beer ${ }^{5,13 凶}$
}

\begin{abstract}
During the evolution of SARS-CoV-2 in humans, a D614G substitution in the spike glycoprotein (S) has emerged; virus containing this substitution has become the predominant circulating variant in the COVID-19 pandemic ${ }^{1}$. However, whether the increasing prevalence of this variant reflects a fitness advantage that improves replication and/or transmission in humans or is merely due to founder effects remains unknown. Here we use isogenic SARS-CoV-2 variants to demonstrate that the variant that contains S(D614G) has enhanced binding to the human cell-surface receptor angiotensin-converting enzyme 2 (ACE2), increased replication in primary human bronchial and nasal airway epithelial cultures as well as in a human ACE2 knock-in mouse model, and markedly increased replication and transmissibility in hamster and ferret models of SARS-CoV-2 infection. Our data show that the D614G substitution in S results in subtle increases in binding and replication in vitro, and provides a real competitive advantage in vivo-particularly during the transmission bottleneck. Our data therefore provide an explanation for the global predominance of the variant that contains S(D614G) among the SARS-CoV-2 viruses that are currently circulating.
\end{abstract}

In late 2019, SARS-CoV-2 was detected in Wuhan (Hubei province, China) ${ }^{2,3}$ and rapidly led to the COVID-19 pandemic; by December 2020,70 million cases and 1.5 million deaths attributable to this disease had been confirmed $^{4}$.SARS-CoV-2 causes a life-threatening pneumonia in vulnerable groups of people ${ }^{5}$. The entry of SARS-CoV-2 into cells is dependent on the interaction of $\mathrm{S}$ and $\mathrm{ACE} 2^{3,6}$. S is a homotrimeric class-I fusion protein that comprises two subunits (S1 and S2) that are separated by a protease cleavage site. S1 forms a globular head and is essential for receptor binding, and S2 mediates fusion of the viral envelope with host cell membranes. During entry, the receptor-binding domain within the $\mathrm{S} 1$ subunit binds ACE2, which generates conformational changes in the S2 subunit and facilitates internalization of the virus ${ }^{7,8}$. S(D614G) is a variant of $S$ that contains a substitution outside of the receptor-binding domain that is thought to cause a conformational change in the protein, which improves ACE2 binding and increases the probability of infection ${ }^{1,9}$.

As the pandemic has progressed, the SARS-CoV-2 variant that contains S(D614G) (hereafter, SARS-CoV-2 $\left.{ }^{\mathrm{G} 614}\right)$ ) has rapidly superseded the parental variant (with $D$ in amino acid position 614 of $S$; hereafter, SARS-CoV-2 ${ }^{\mathrm{D} 614}$ ) in frequency to become globally dominant. Such a shift in genotype frequency might be caused by a founder effect following introduction into a highly interconnected population; alternatively, SARS-CoV-2 ${ }^{\mathrm{G} 614}$ may have a fitness advantage over SARS-CoV- $2^{\mathrm{D} 614}$. Some studies have suggested the D614G substitution in S may confer a fitness advantage on the virus by improving cell entry ${ }^{8,9}$. To address the role of D614G substitution in S in the dissemination and predominance of SARS-CoV-2 ${ }^{\mathrm{G} 614}$ during the COVID-19 pandemic, we characterized S binding to human ACE2 (hACE2) and replication kinetics in vitro, and evaluated infection and transmission dynamics in vivo using three animal models. Our data show that the D614G substitution in S confers increased binding to the hACE2 receptor and increased replication in primary human airway epithelial cultures. Moreover, comparison of recombinant isogenic SARS-CoV-2 variants demonstrates that the D614G substitution in S provides competitive advantage in a hACE2 knock-in mouse model, and markedly increases replication and transmission in Syrian hamster and ferret models of SARS-CoV-2 infection.

\section{D614G substitution in S enhances hACE2 binding}

To measure the effects of the D614G substitution in S, we first quantified the binding of S1 domain monomers to hACE2 using biolayer

${ }^{1}$ CDC COVID-19 Response, Centers for Disease Control and Prevention, Atlanta, GA, USA. ${ }^{2}$ Institute of Virology and Immunology (IVI), Mittelhäusern, Switzerland. ${ }^{3}$ Department of Infectious Diseases and Pathobiology, Vetsuisse Faculty, University of Bern, Bern, Switzerland. ${ }^{4}$ Graduate School for Biomedical Science, University of Bern, Bern, Switzerland. ${ }^{5}$ Institute of Diagnostic

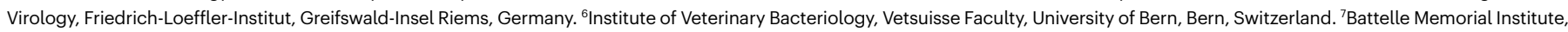
Atlanta, GA, USA. ${ }^{8}$ Institute of Cell Biology, University of Bern, Bern, Switzerland. ${ }^{9} \mathrm{COMPATH}$, Institute of Animal Pathology, University of Bern, Bern, Switzerland. ${ }^{10}$ Oak Ridge Institute for

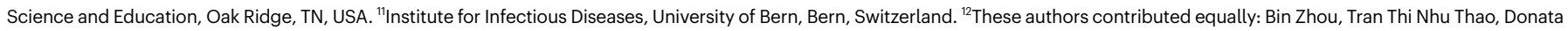
Hoffmann, Adriano Taddeo. ${ }^{13}$ These authors jointly supervised this work: Charaf Benarafa, David E. Wentworth, Volker Thiel, Martin Beer. ${ }^{凶}$-mail: charaf.benarafa@vetsuisse.unibe.ch; dwentworth@cdc.gov; volker.thiel@vetsuisse.unibe.ch; martin.beer@fli.de 


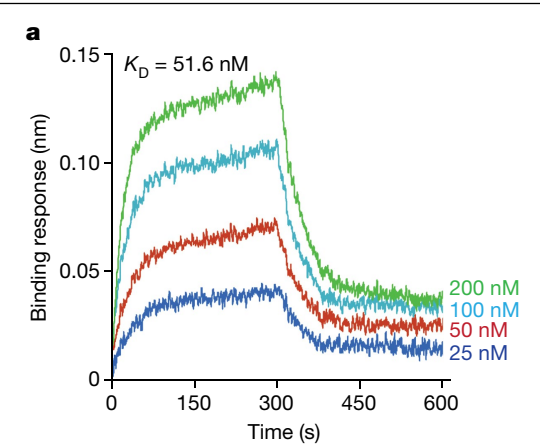

b

Concentration $\quad \mathrm{S} 1-\mathrm{His} \quad \mathrm{S} 1-\mathrm{Fc}$

(ng per $10^{6}$ cells) fluorescent signal fluorescent signal
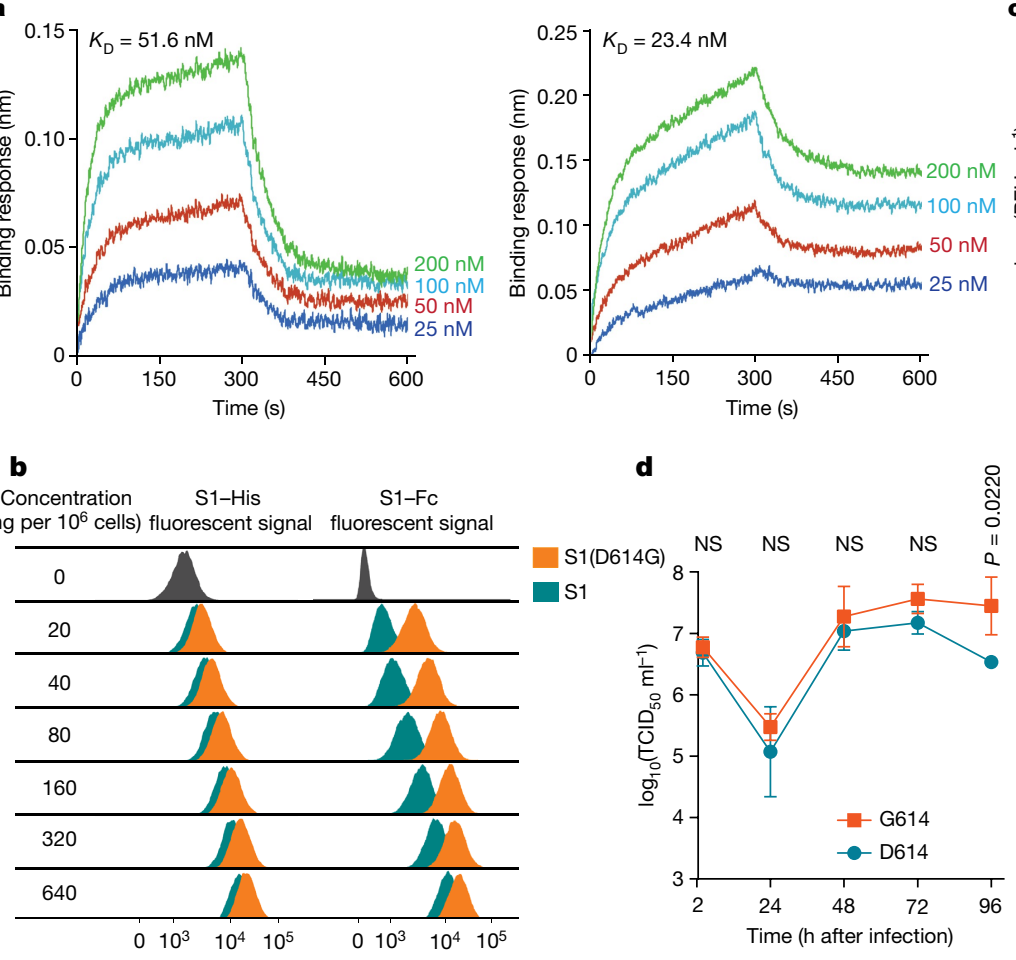

d
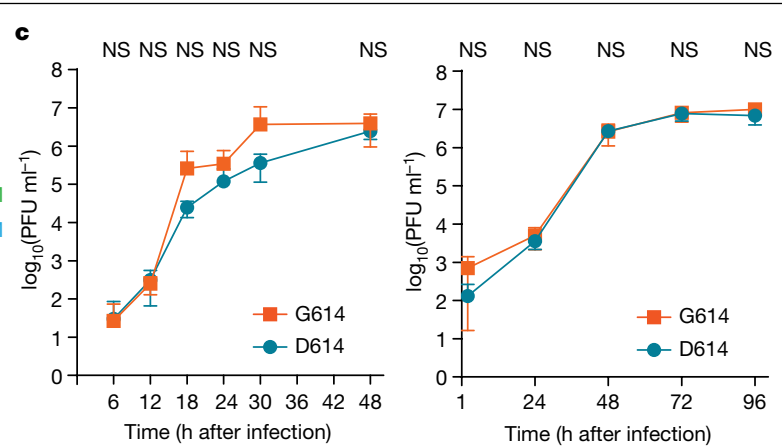

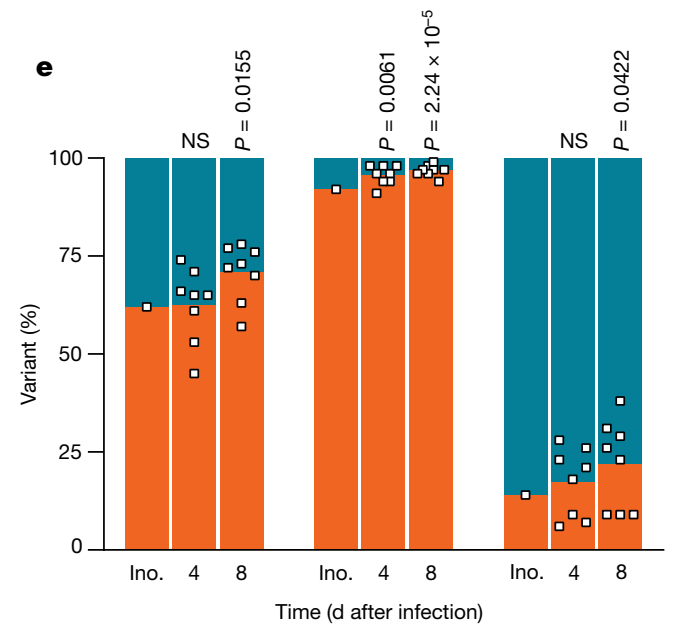

Fig. 1 In vitro characterization of S1 and of recombinant SARS-CoV-2 ${ }^{\text {D614 }}$ and SARS-CoV-2 ${ }^{\mathrm{G} 614}$. a, Affinity between $\mathrm{S} 1$ and hACE2 determined by biolayer interferometry. Fc-tagged ACE2 protein was loaded onto surface of anti-human Fc capture biosensors. Association was conducted using S1 (left) or S1(D614G) (right), followed by dissociation. Data represent three biological replicates.

b, Binding of polyhistidine-tagged or Fc-tagged S1 to BHK-hACE2 cells is shown as peaks of fluorescence, detected by flow cytometry. c, Replication kinetics of recombinant viruses in Vero $\mathrm{E} 6$ cells at $37^{\circ} \mathrm{C}$ (left) and human nasal epithelial cells at $33^{\circ} \mathrm{C}$ (right). Supernatant was collected at the indicated time points and titrated by plaque assay. D614, SARS-CoV-2 ${ }^{\mathrm{D} 614} ; \mathrm{G} 614, \mathrm{SARS}-\mathrm{CoV}-2^{\mathrm{G} 614}$.

d, Replication kinetics of recombinant viruses in human NBE cells at $33^{\circ} \mathrm{C}$ (left), $37^{\circ} \mathrm{C}$ (middle) and $39^{\circ} \mathrm{C}$ (right). Supernatant was collected daily and titrated by $\mathrm{TCID}_{50}$ assay. In c, d, data are mean \pm s.d. of three biological replicates (Vero E6 cells) or four technical replicates (human nasal epithelial and human NBE cells).

interferometry. Both S1 and S1(D614G) bind efficiently to hACE2; however, S1(D614G) showed an affinity about twofold higher than that of S1 (Fig. 1a, Supplementary Table 2). Similarly, S(D614G) had a higher affinity to hACE2 than that of S when full-length monomeric forms were used (Extended Data Fig. 1a, Supplementary Table 2). The D614G substitution in S also resulted in enhanced S1 binding to hACE2 exogenously expressed in baby hamster kidney cells (hereafter, BHK-hACE2 cells)
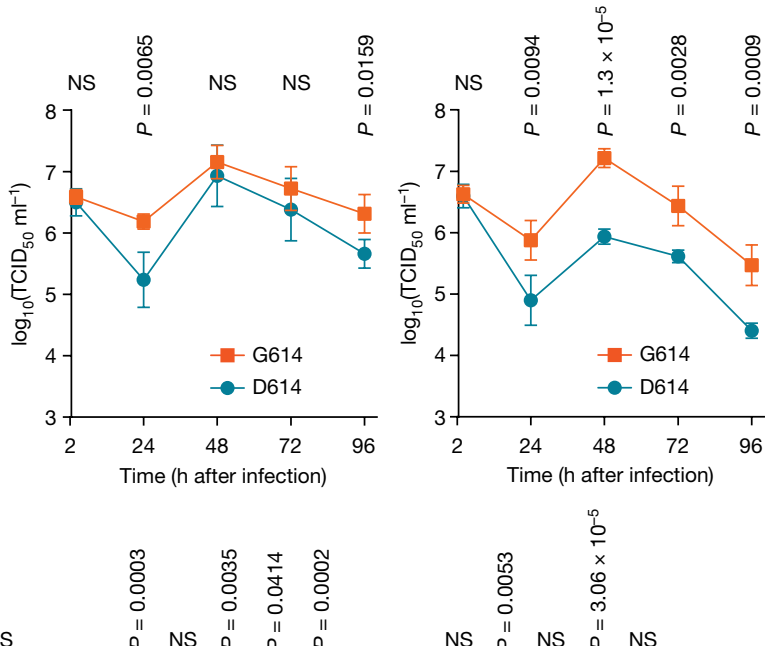

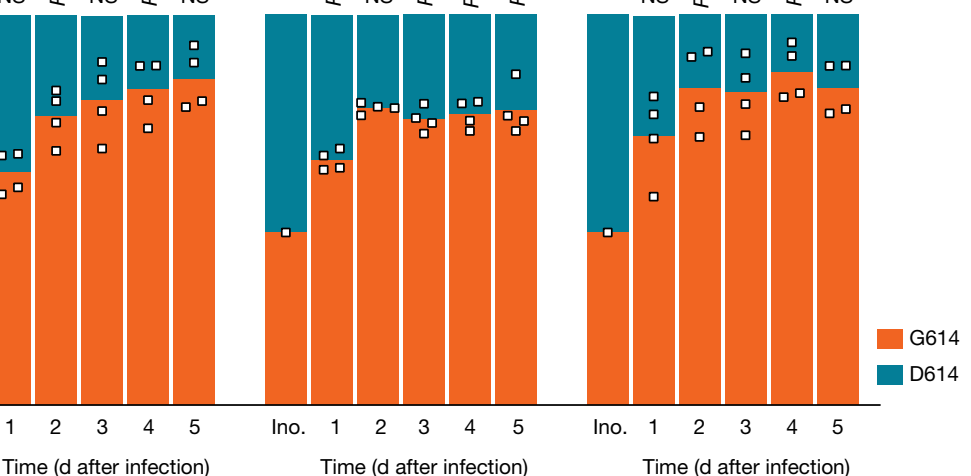

Statistical significance was determined by two-sided, unpaired Student's $t$-test without adjustments for multiple comparisons. e, f, Competition assay of recombinant viruses in human nasal epithelial cells at $33^{\circ} \mathrm{C}(\mathbf{e})$ and human NBE cells at $33^{\circ} \mathrm{C}\left(\mathbf{f}\right.$, left), $37^{\circ} \mathrm{C}\left(\mathbf{f}\right.$, middle) and $39^{\circ} \mathrm{C}(\mathbf{f}$, right). The inoculum (ino.) was prepared by mixing two viruses and used for infection of human nasal epithelial (1:1 (e, left), 1:10 (e, middle) and 10:1 (e, right) PFU ratios, 8 technical replicates each) and human NBE (1:1 PFU ratio, 4 technical replicates each) cells. Apical wash and supernatant were collected daily, and extracted RNA was used for NGS. Bar graphs show percentage of sequence reads encoding $S$ or $\mathrm{S}$ (D614G). In competition experiments in human nasal epithelial and human NBE cells, each square represents an individual data point. For each time point, a linear regression model was generated on the basis of the sequencing read counts for S and S(D614G), and $P$ values were calculated for the group (variant) coefficient. NS, not significant $(P>0.05)$.

(Fig. 1b, Extended Data Fig. 1b). The binding of polyhistidine-tagged S1 or S1(D614G) to BHK-hACE2 cells showed that more S1(D614G) bound to BHK-hACE2 cells than did S1, by flow cytometry (Fig. 1b, Extended Data Fig. 1b). When using homodimeric recombinant constructs comprising S1 attached to an IgG C terminus, we observed a more notable effect in the increased binding of the S1(D614G) protein to the BHK-hACE2 cell (Fig. 1b, Extended Data Fig. 1b). 


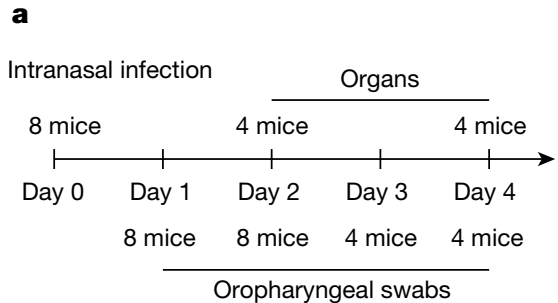

c

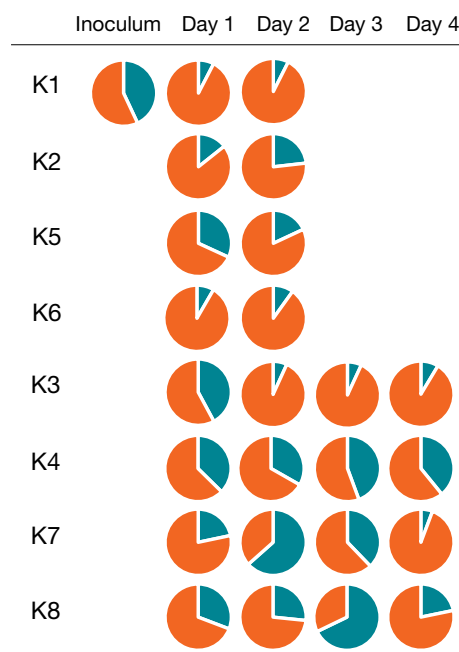

Fig. 2 | Replication of SARS-CoV-2 ${ }^{\mathrm{D} 614}$ and SARS-CoV-2 ${ }^{\mathrm{G} 614}$ in hACE2 knock-in mice. a, Experimental scheme for intranasal infection of hACE2 knock-in mice with recombinant SARS-CoV-2 ${ }^{\mathrm{D} 614}$ and SARS-CoV- $2^{\mathrm{G} 614}$. Oropharyngeal swabs were sampled daily and tissue samples were analysed in subgroups of 4 mice at 2 and 4 days after infection in 2 independent experiments. b, Quantitative PCR with reverse transcription (RT-qPCR) analysis of oropharyngeal swabs of

\section{SARS-CoV-2 ${ }^{\mathrm{G} 614}$ replication in epithelial cells}

To assess the effect of S(D614G) in the context of virus infection, we generated an isogenic SARS-CoV-2 ${ }^{\mathrm{D} 614}$ and SARS-CoV-2 ${ }^{\mathrm{G} 614}$ pair using a SARS-CoV-2 reverse genetics system ${ }^{10}$. The molecular clone based on the Wuhan-Hu-1 isolate is representative of SARS-CoV-2 ${ }^{\mathrm{D} 614}$ (refs. ${ }^{10,11}$ ). The sequence of the isogenic SARS-CoV- $2^{\mathrm{G} 614}$ was engineered with an A-to-G transition at position 23,403 to encode a glycine at position 614 of S. The identity of the resulting recombinant SARS-CoV- $-2^{\mathrm{G} 614}$ was confirmed by genomic next-generation sequencing (NGS) of virus stock (passage 1) used in subsequent experiments. The replication kinetics of SARS-CoV-2 ${ }^{\mathrm{D} 614}$ and SARS-CoV-2 ${ }^{\mathrm{G} 614}$ in Vero E6 cells differed only marginally (Fig. 1c). We assessed replication kinetics in primary human nasal epithelial and primary normal human bronchial epithelial (human NBE) cell cultures grown under air-liquid interface conditions that resemble the pseudostratified epithelial lining of the human respiratory epithelium. We observed no significant difference in the primary human nasal epithelial cells after infection of SARS-CoV-2 ${ }^{\mathrm{D} 614}$ or SARS-CoV-2 ${ }^{\mathrm{G} 614}$ at $33^{\circ} \mathrm{C}$ (the temperature of the nasal epithelium) (Fig.1C). By contrast, SARS-CoV-2 ${ }^{\mathrm{G} 614}$ displayed elevated titres in human NBE cells at $33^{\circ} \mathrm{C}, 37^{\circ} \mathrm{C}$ and $39^{\circ} \mathrm{C}$, which mimic the temperatures of the upper respiratory tract, lower respiratory tract and fever, respectively (Fig.1d). Infection kinetics of human NBE cells with the natural isolates SARS-CoV-2/USA-WA1/2020 (SARS-CoV-2 ${ }^{\text {D614 }}$ ) or SARS-CoV-2/Massachusetts/VPT1/2020 (SARS-CoV-2 ${ }^{\mathrm{G} 614}$ ) showed a more subtle advantage for SARS-CoV- $2^{\mathrm{G} 614}$ at $37^{\circ} \mathrm{C}$ and $39^{\circ} \mathrm{C}$ (Extended Data Fig. 1c). To refine

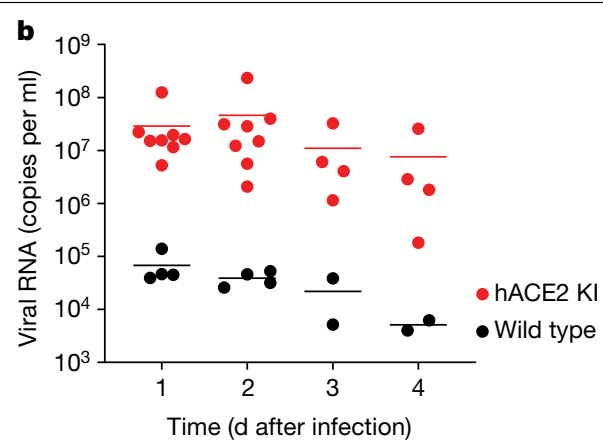

d

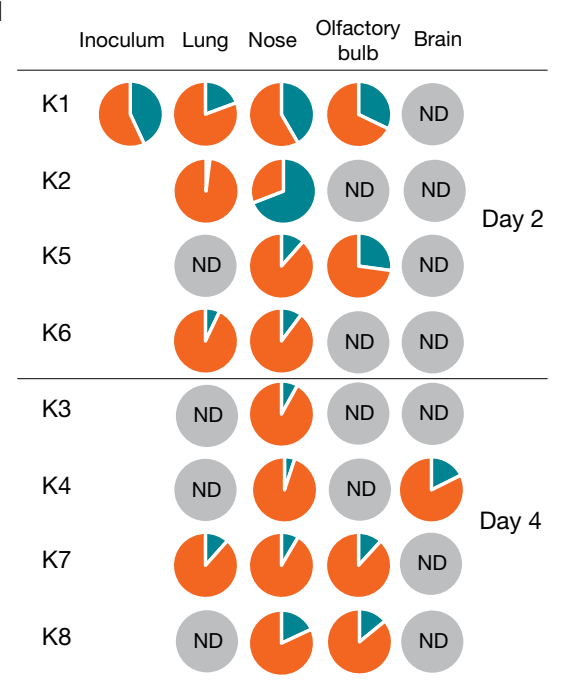

inoculated hACE2 knock-in (KI) and wild-type mice. c, d, Pie chart of the mean frequencies of the $A$ or $G$ nucleotide at position 23,403 (corresponding to $D$ (in blue) or $\mathrm{G}$ (in orange) at amino acid position 614, respectively). K1 to K8 denote individual mice. Each pie chart illustrates the ratio of $A / G$ detected from individual oropharyngeal swab samples (c) and tissues (d) at the indicated time after infection. ND, not detected.

this analysis, we performed competition experiments by infecting human nasal epithelial and human NBE cell cultures with a mixture of isogenic SARS-CoV-2 ${ }^{\mathrm{D} 614}$ and SARS-CoV-2 ${ }^{\mathrm{G} 614}$ at defined ratios. In both of the primary human respiratory cell culture systems, the ratio of SARS-CoV-2 ${ }^{\mathrm{G} 614}$ to SARS-CoV-2 ${ }^{\mathrm{D} 614}$ shifted in favour of SARS-CoV-2 ${ }^{\mathrm{G} 614}$ across five or eight days of infection (Fig. 1e, f, Extended Data Figs. 1d, e, 2a, b). Collectively, these results show that the D614G substitution in $\mathrm{S}$ is associated with enhanced hACE2 binding and increased replication in primary human airway epithelial cell models of SARS-CoV-2 infection.

\section{SARS-CoV-2 ${ }^{\mathrm{G} 614}$ replication in humanized $A C E 2$ mice}

Mice do not support the efficient replication of SARS-CoV-2 unless they are genetically engineered to express hACE $2^{12,13}$. To evaluate the relative fitness of SARS-CoV-2 ${ }^{\mathrm{G} 614}$ in vivo, we generated knock-in mice that express human $A C E 2$ under the endogenous regulatory elements of mouse Ace2 (Extended Data Fig. 3a). We intranasally inoculated eight heterozygous female mice with an equal mixture of both viruses (SARS-CoV-2 ${ }^{\mathrm{D} 614}$ and SARS-CoV-2 ${ }^{\mathrm{G} 614}$ ) in a competition experiment, by pooling $1 \times 10^{5}$ plaque-forming units (PFU) of each variant (Fig. $2 \mathrm{a}$ ). We monitored viral RNA loads in oropharyngeal swabs daily, and in various tissues (at days 2 and 4 after inoculation) by real-time PCR. We did not observe any significant loss of body weight in hACE2 knock-in mice up to day 4 after inoculation (Extended Data Fig. 3b). Our longitudinal analysis of oropharyngeal swabs revealed efficient virus replication in 


\begin{tabular}{|c|c|c|c|c|c|c|c|c|c|}
\hline G614 & \multicolumn{9}{|c|}{ Time (d after infection) } \\
\hline D614 & Inoculum & 2 & 3 & 4 & 5 & 6 & 7 & 8 & 12 \\
\hline
\end{tabular}

Intranasally infected

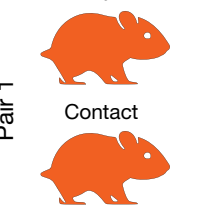

12
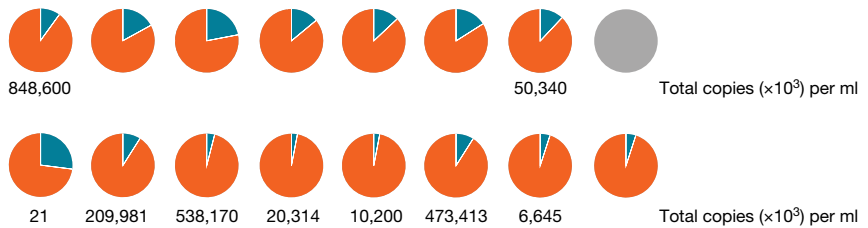

Intranasally infected
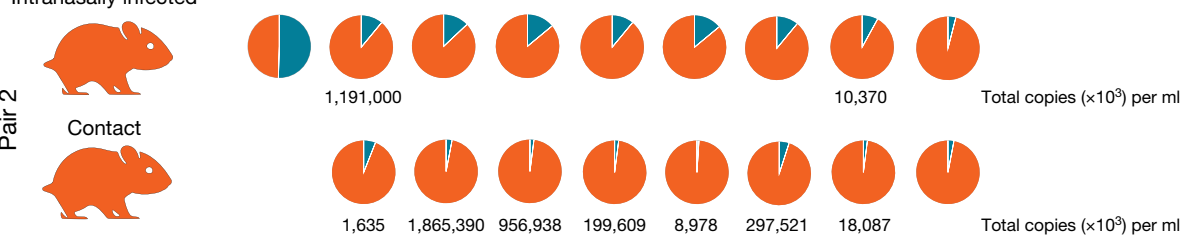

Intranasally infected
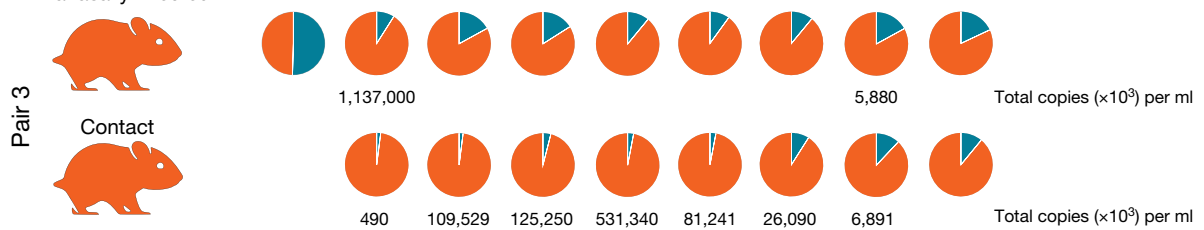

Intranasally infected
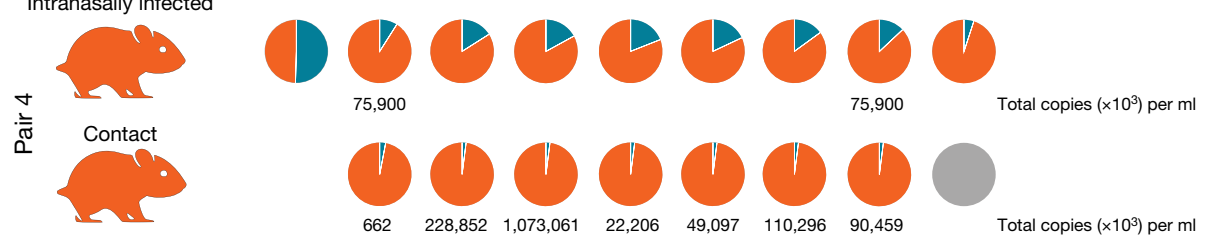

Intranasally infected
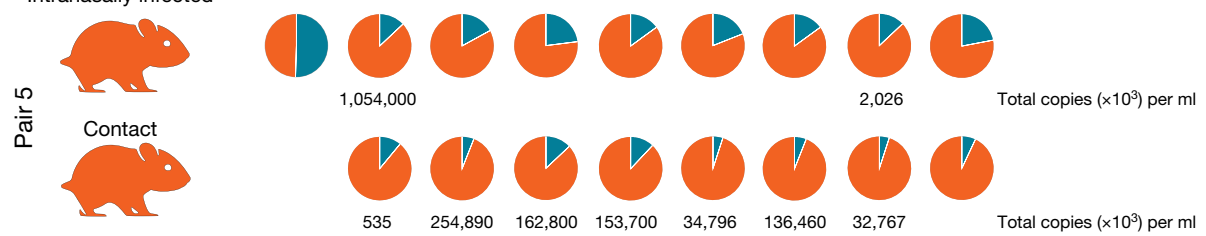

Total copies $\left(\times 10^{3}\right)$ per $\mathrm{ml}$

Intranasally infected
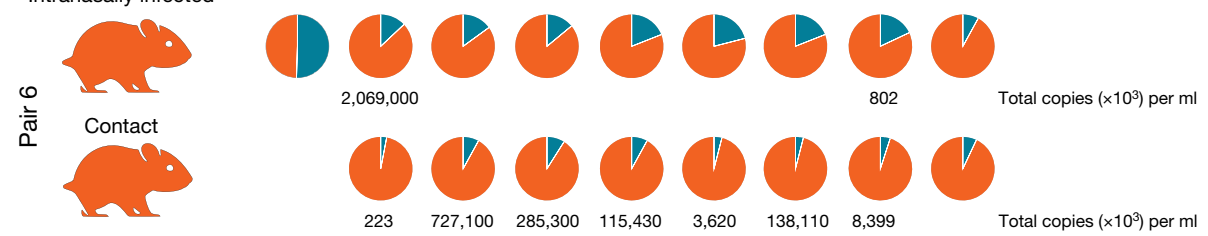

Fig. 3 | Replication and transmission of SARS-CoV-2 ${ }^{\mathrm{D} 614}$ and SARS-CoV-2 ${ }^{\mathrm{C} 614}$ in Syrian hamsters. The transmission of SARS-CoV-2 ${ }^{\mathrm{D} 614}$ and SARS-CoV-2 ${ }^{\mathrm{G} 614}$ by hamsters in a pairwise one-by-one setup with direct contact of donor and cohoused contact hamsters is illustrated. Samples of nasal washings were taken daily between day 2 and day 8 after infection (dpi), and finally at 12 days after infection, and were then analysed. Pie chart of the fraction of A or G nucleotide at position 23,403 (corresponding to D or $G$ at amino acid position 614, respectively), measured by NGS of amplicons. Genome copies were calculated from RT-qPCR using an RNA standard. Orange colouring of the hamster silhouette refers to the detection of $\mathrm{G}$ (that is, SARS-CoV-2 ${ }^{\mathrm{G} 614}$ ) and blue to the detection of A (that is, SARS-CoV-2 ${ }^{\mathrm{D} 614}$ ) at most time points. Grey colouring, no infection detected. the upper respiratory tract of hACE2 knock-in mice relative to wild-type mice (Fig. 2b). Accordingly, tissue samples collected at days 2 and 4 after inoculation revealed high viral loads in the nasal conchae, lungs and olfactory bulbs, and lower levels in the brain (Extended Data Fig.3c, d). Levels of viral RNA were low to undetectable in spleen, small intestine, kidneys and faeces (data not shown). We did not find any overt pathological lesions in the lungs at days 2 and 4 after inoculation (Supplementary Table 3,4). NGS of the oropharyngeal swabs revealed a net advantage for SARS-CoV-2 ${ }^{\mathrm{G} 614}$ over SARS-CoV-2 ${ }^{\mathrm{D} 614}$ in most mice and at most time points (Fig. 2c, Extended Data Fig. 3e, f). We found a similar replication advantage for SARS-CoV-2 ${ }^{\mathrm{G} 614}$ in the organs (Fig. 2d). Collectively, these results demonstrate increased replication of SARS-CoV-2 ${ }^{\mathrm{G} 614}$ in a mouse model that expresses authentic human ACE2.

\section{SARS-CoV-2 ${ }^{6614}$ infection in hamsters and ferrets}

Hamsters are highly susceptible toSARS-CoV-2 infection and develop disease that closely resembles pan-respiratory, moderate-to-severe COVID19 in humans ${ }^{14-16}$. To examine the replication kinetics of the SARS-CoV-2 variants using a hamster model, we intranasally inoculated seven hamsters with SARS-CoV-2 ${ }^{\mathrm{D} 614}$ or SARS-CoV-2 ${ }^{\mathrm{G} 614}\left(1 \times 10^{5.1}\right.$ tissue culture infectious dose $\left(\mathrm{TCID}_{50}\right)$ per hamster, and $10^{4.5} \mathrm{TCID}_{50}$ per hamster, respectively, 

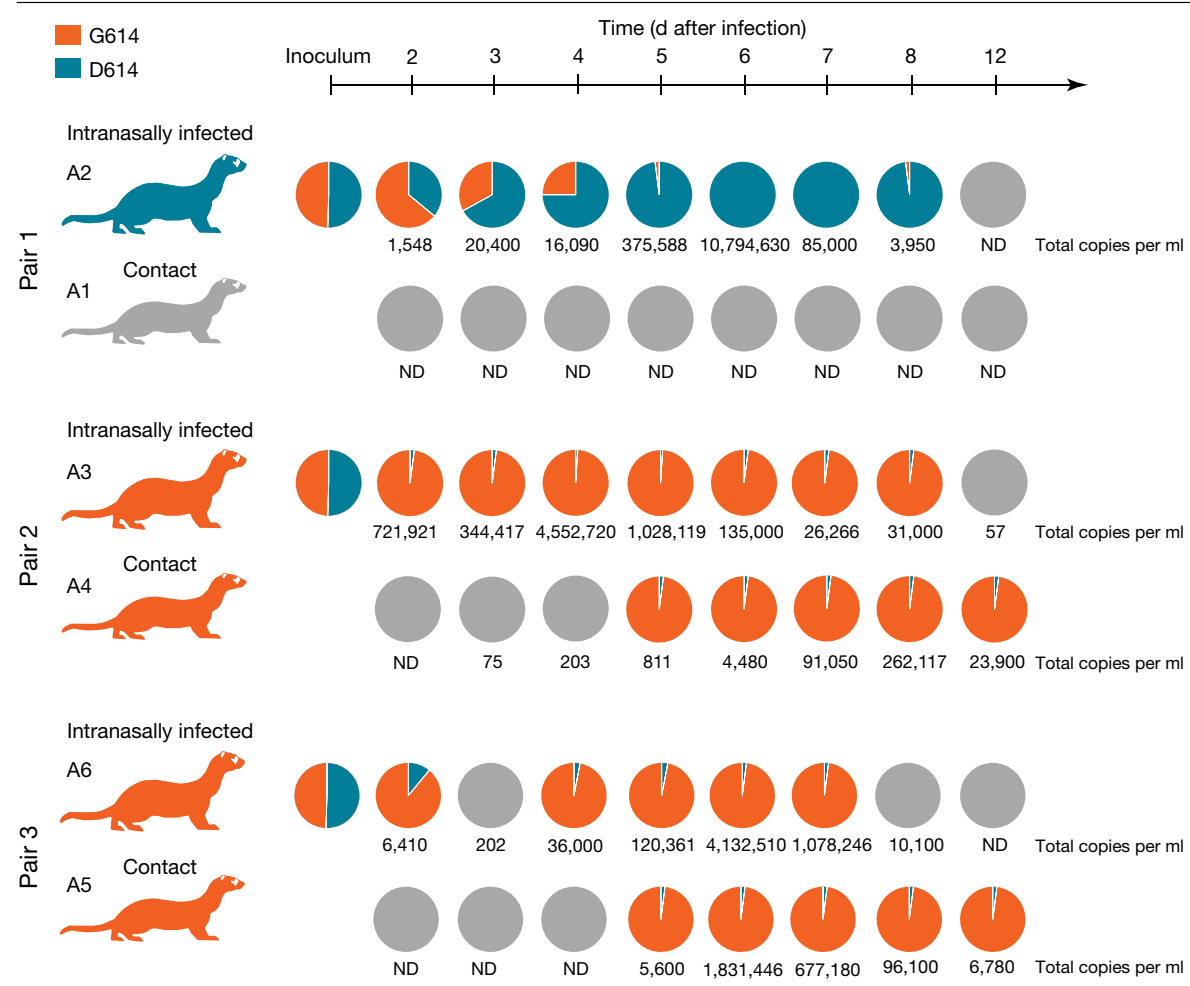

6,780 Total copies per $\mathrm{ml}$
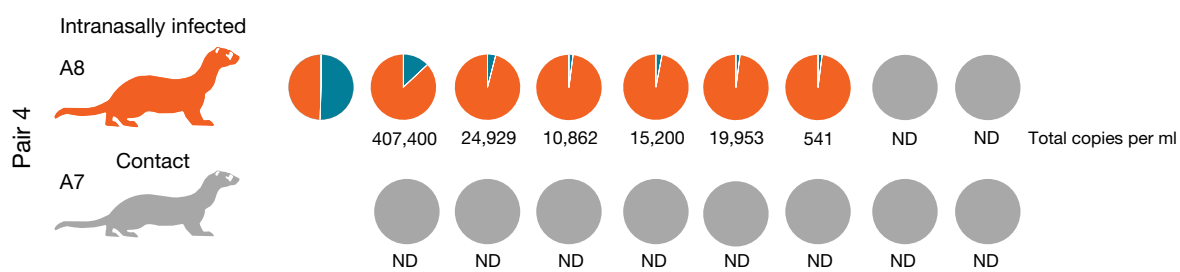

ND
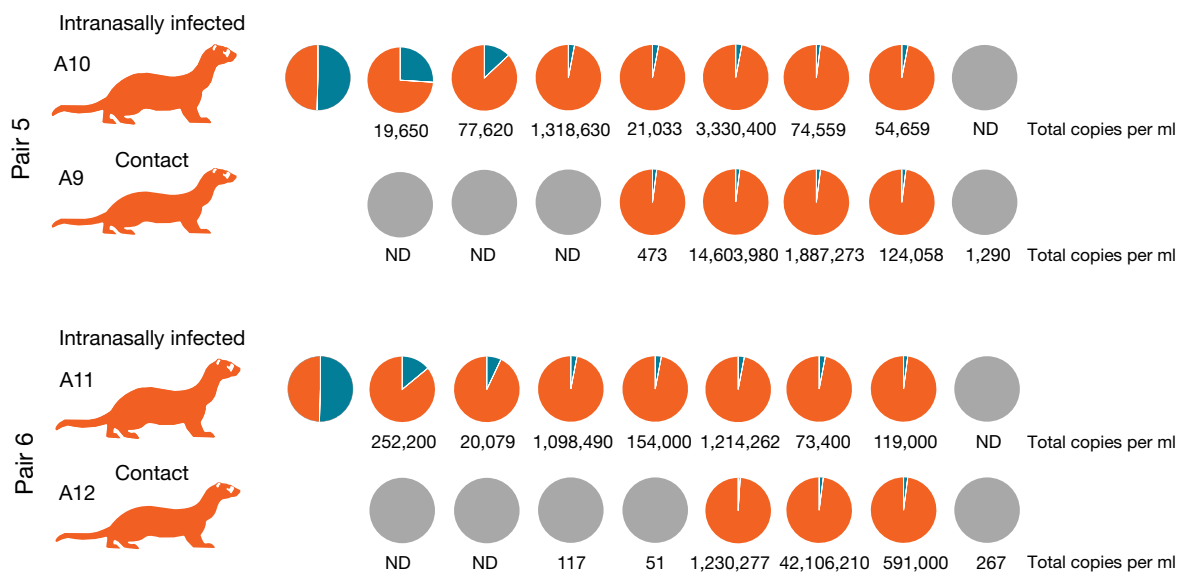

ND Total copies per $\mathrm{ml}$
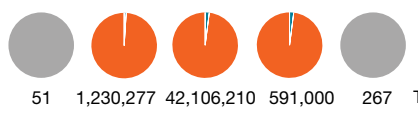

267 Total copies per $\mathrm{ml}$

calculated from back titration of the inoculum) and monitored them for four consecutive days. We did not observe any marked differences in body weight, titres of shed virus or viral loads in respiratory tract tissue between the two groups in the acute phase (Extended Data Fig. $4 \mathrm{c}-\mathrm{e}$ ). For both variants, the highest genome loads were found in the nasal conchae followed by the pulmonary tissue (Extended Data Fig. 4e). These observations suggest that the D614G substitution in S does not have strong effect on clinical outcomes or a detectable replication advantage in infections with SARS-CoV-2 ${ }^{\mathrm{D} 614}$ versus SARS-CoV-2 ${ }^{\mathrm{G} 614}$ in the hamster model.

We therefore set up an in vivo competition experiment to better elucidate the potential replication and/or transmission differences between the variants. We intranasally inoculated six donor Syrian hamsters with
Fig. 4 | Replication and transmission of SARS-CoV-2 ${ }^{\mathrm{D} 614}$ and SARS-CoV-2 ${ }^{\mathrm{G} 614}$ in ferrets. Schematic of the experimental setup, in which six pairs of one donor ferret cohoused with one naive contact ferret were used. Samples of nasal washings were taken daily between day 2 and day 8 after infection, and finally at 12 days after infection, and were then analysed. Pie chart of fraction of $\mathrm{A}$ or $\mathrm{G}$ nucleotide at position 23,403 (corresponding to $\mathrm{D}$ or $\mathrm{G}$ at amino acid position 614 , respectively). Each pie chart illustrates the ratio of $\mathrm{A} / \mathrm{G}$ detected from individual nasal washing samples over time. Orange colouring of the ferret silhouette refers to the detection of $G$ (that is, SARS-CoV-2 ${ }^{\mathrm{G} 614}$ ) at most time points, and blue indicates detection of A (that is, SARS-CoV$\left.2^{\mathrm{D} 614}\right)$. Numbers represent total genome copies per ml. Grey, no infection or viral genome number too low to determine the $\mathrm{A} / \mathrm{G}$ ratio by NGS. a 1:1 mixture (on the basis of infectious PFU titre) of SARS-CoV-2 ${ }^{\mathrm{D} 614}$ and SARS-CoV-2 ${ }^{\mathrm{G} 614}$ in direct 'one-to-one' transmission experiments. We later confirmed the RNA ratio of the two variants within the inoculum using NGS, and absolute quantification using digital-droplet PCR with allele-specific locked nucleic acid probes (Fig. 3). At $24 \mathrm{~h}$ after inoculation, each inoculated hamster was cohoused with a naive hamster. Viral RNA load in daily nasal washings, changes in body weight and clinical signs all agreed with previously published data ${ }^{14-16}$ (Extended Data Fig. 4a, b). NGS of the viral RNA sequence composition of nasal washings revealed a prominent shift towards SARS-CoV-2 ${ }^{\mathrm{G} 614}$ within 48 h of inoculation (Fig. 3 , Extended Data Fig. 4f, g). The transmission efficiency was $100 \%$, and analysis of the RNA sequence composition showed that SARS-CoV-2 ${ }^{\mathrm{G} 614}$ 
represented over $90 \%$ of the viral RNA in the contact hamsters (Fig. 3). In summary, at $24 \mathrm{~h}$ after inoculation hamsters inoculated with an equal ratio of SARS-CoV-2 ${ }^{\mathrm{D} 614}$ and SARS-CoV-2 ${ }^{\mathrm{G} 614}$ transmit primarily SARS-CoV-2 ${ }^{\mathrm{G} 614}$.

We further investigated the competitive advantage of S(D614G) in ferrets, which represent a good model of SARS-CoV-2 transmission ${ }^{17}$ in which the virus replicates primarily within the upper respiratory tract (resembling mild infections in humans). We intranasally inoculated six ferrets with a 1:1 mixture (on the basis of infectious PFU titre) of SARS-CoV-2 ${ }^{\mathrm{D} 614}$ and SARS-CoV-2 ${ }^{\mathrm{G} 614}$ in direct one-to-one transmission experiments. For all six inoculated ferrets, SARS-CoV-2-infection was confirmed: body weight changes and viral RNA loads in nasal washings (Fig. 4, Extended Data Fig. 5a,b) were consistent with previously published data $^{17,18}$. In five of the six inoculated ferrets, SARS-CoV- $2^{\mathrm{G} 614}$ became the dominant variant (Fig. 4, Extended DataFig. 5c, d). In addition, SARS-CoV-2 transmission occurred in four of the six ferret pairs. In each pair with successful transmission, SARS-CoV-2 ${ }^{\mathrm{G} 614}$ prevailed over SARS-CoV-2 ${ }^{\mathrm{D} 614}$ (Fig. 4). All NGS data from the ferret samples were retested by absolute quantification using allele-specific locked nucleic acid probes and digital droplet PCR analysis. Notably, the inoculated ferret from pair 1 (in which SARS-CoV-2 ${ }^{\mathrm{D} 614}$ predominated the viral population) did not transmit virus to the contact, despite a high peak viral genome load of more than 10 million copies per ml. By contrast, the lack of transmission in pair 4 (in which SARS-CoV- $2^{\mathrm{G} 614}$ became the dominant variant) was connected to peak viral loads of below 500,000 genome copies per ml (Fig. 4). In summary, the competition experiment in ferrets revealed that SARS-CoV-2 ${ }^{\mathrm{G} 614}$ preferentially infected and replicated in five out of six inoculated ferrets, and the successful transmission events occurred only with SARS-CoV- ${ }^{\mathrm{G} 614}$.

\section{Discussion}

The evolution of SARS-CoV-2 in humans has been proposed to be a nondeterministic process in which virus diversification results mainly from random genetic drift, suggesting no strong selective pressure is acting on its adaptation in humans ${ }^{19}$. However, since the emergence of the D614G substitution in S in early 2020, the SARS-CoV- ${ }^{\mathrm{G} 614}$ variant has become globally prevalent ${ }^{1}$. Both a founder effect and structural changes to $S$ have previously been proposed as driving forces in establishing the prevalence of SARS-CoV- $2^{\mathrm{G} 614}$. Previous structural studies and the use of pseudotyped viruses have suggested that SARS-CoV-2 ${ }^{\mathrm{G} 614}$ may confer increased infectivity as a result of an increased 'open' conformation of the receptor-binding domain for receptor binding or increased S stability ${ }^{1,9}$. In contrast to these studies (which used recombinant trimeric S), we used a reductionist approach to eliminate complications due to 'open' or 'closed' conformations of the receptor-binding domain in trimeric S. We found that S1(D614G) or monomeric S(D614G) both had increased affinity to hACE2, which may be another mechanism that underlies the increased replication and transmission of SARS-CoV- ${ }^{\mathrm{G} 614}$.

Studies using isogenic SARS-CoV- $2^{\mathrm{D} 614}$ and SARS-CoV- $2^{\mathrm{G} 614}$ to assess the phenotype in the context of SARS-CoV-2 infection have recently been reported ${ }^{20,21}$. Both of these studies have demonstrated that SARS-CoV- ${ }^{\mathrm{G} 614}$ increased replication in vitro, and one $\mathrm{e}^{21}$ observed earlier transmission in a hamster model. We extended these studies by exploiting a range of in vitro and in vivo models of SARS-CoV-2 infection: primary human nasal epithelial and human NBE cell cultures, a hACE2 knock-in mouse model, a hamster model and a ferret model. Throughout these experimental systems, we consistently observed an increased replicative fitness of SARS-CoV- ${ }^{\mathrm{G} 614}$ over SARS-CoV-2 ${ }^{\mathrm{D} 614}$, by applying NGS techniques and allele-specific absolute quantification for confirmation. The advantage provided by the D614G substitution in S was most prominent in competition and transmission experiments in hamsters and ferrets, and must therefore be considered as a driving force that has led to the global dominance of SARS-CoV- ${ }^{\mathrm{G} 614}$.

Our data also agree with previously reported functional changes conferred by the D614G substitution in $\mathrm{S}^{1}$, and with previously reported increased infectivity of pseudotyped viruses containing this substitution ${ }^{9,22}$. Although our studies establish a phenotype of increased replication and transmission of SARS-CoV-2 ${ }^{\mathrm{G} 614}$, there is no evidence for a change in pathogenicity in any of the animal models we used. This is important because infection with SARS-CoV- $2^{\mathrm{G} 614}$ is not associated with the development of severe COVID-19 in humans ${ }^{1}$.

The ongoing pandemic will probably give rise to additional variants of SARS-CoV-2 that may display phenotypic changes and further adaptations to human or animal (such as mink) reservoirs. The ability to rapidly identify emerging variants using genomics, reconstructing emerging virus variants and assessing their phenotypes in vitro and in vivo will allow a rapid response to their emergence with appropriate countermeasures. The mouse model described in this Article, which is based on hACE2 expression under the endogenous regulatory elements of mouse Ace2, is a valuable tool and will complement existing animal models of SARS-CoV-2 infection. Similar to our demonstration here of the increased replication and transmissibility of SARS-CoV- $2^{\mathrm{G} 614}$, the phenotypic assessment of future variants will probably require several complementary animal models that together reflect aspects of SARS-CoV-2 replication, transmission and pathogenicity in humans.

\section{Online content}

Any methods, additional references, Nature Research reporting summaries, source data, extended data, supplementary information, acknowledgements, peer review information; details of author contributions and competing interests; and statements of data and code availability are available at https://doi.org/10.1038/s41586-021-03361-1.

1. Korber, B. et al. Tracking changes in SARS-CoV-2 spike: evidence that D614G increases infectivity of the COVID-19 virus. Cell 182, 812-827.e19 (2020).

2. Zhu, N. et al. A novel coronavirus from patients with pneumonia in china, 2019. N. Engl. J. Med. 382, 727-733 (2020).

3. Zhou, P. et al. A pneumonia outbreak associated with a new coronavirus of probable bat origin. Nature 579, 270-273 (2020)

4. ECDC. Communicable disease threats report, week 51, 13-19 December 2020. https:// www.ecdc.europa.eu/sites/default/files/documents/Communicable-disease-threatsreport-19-dec-2020.pdf (2020).

5. Huang, C. et al. Clinical features of patients infected with 2019 novel coronavirus in Wuhan, China. Lancet 395, 497-506 (2020).

6. Letko, M., Marzi, A. \& Munster, V. Functional assessment of cell entry and receptor usage for SARS-CoV-2 and other lineage B betacoronaviruses. Nat. Microbiol. 5, 562-569 (2020).

7. Wrapp, D. et al. Cryo-EM structure of the $2019-\mathrm{nCoV}$ spike in the prefusion conformation. Science 367, 1260-1263 (2020).

8. Hoffmann, M. et al. SARS-CoV-2 cell entry depends on ACE2 and TMPRSS2 and is blocked by a clinically proven protease inhibitor. Cell 181, 271-280.e8 (2020).

9. Yurkovetskiy, L. et al. Structural and functional analysis of the D614G SARS-CoV-2 spike protein variant. Cell 183, 739-751.e8 (2020).

10. Thi Nhu Thao, T. et al. Rapid reconstruction of SARS-CoV-2 using a synthetic genomics platform. Nature 582, 561-565 (2020).

11. Wu, F. et al. A new coronavirus associated with human respiratory disease in China. Nature 579, 265-269 (2020)

12. Bao, L. et al. The pathogenicity of SARS-CoV-2 in hACE2 transgenic mice. Nature 583, 830-833 (2020).

13. Jiang, R.D. et al. Pathogenesis of SARS-CoV-2 in transgenic mice expressing human angiotensin-converting enzyme 2. Cell 182, 50-58.e8 (2020).

14. Sia, S. F. et al. Pathogenesis and transmission of SARS-CoV-2 in golden hamsters. Nature 583, 834-838 (2020).

15. Osterrieder, N. et al. Age-dependent progression of SARS-CoV-2 infection in Syrian hamsters. Viruses 12, 779 (2020).

16. Imai, M. et al. Syrian hamsters as a small animal model for SARS-CoV-2 infection and countermeasure development. Proc. Natl Acad. Sci. USA 117, 16587-16595 (2020).

17. Richard, M. et al. SARS-CoV-2 is transmitted via contact and via the air between ferrets. Nat. Commun. 11, 3496 (2020).

18. Kim, Y. I. et al. Infection and rapid transmission of SARS-CoV-2 in ferrets. Cell Host Microbe 27, 704-709.e2 (2020).

19. Alouane, T. et al. Genomic diversity and hotspot mutations in 30,983 SARS-CoV-2 genomes: moving toward a universal vaccine for the "confined virus"? Pathogens 9, 829 (2020).

20. Plante, J. A. et al. Spike mutation D614G alters SARS-CoV-2 fitness. Nature https://doi. org/10.1038/s41586-020-2895-3 (2020).

21. Hou, Y. J. et al. SARS-CoV-2 D614G variant exhibits efficient replication ex vivo and transmission in vivo. Science 370, 1464-1468 (2020).

22. Li, Q. et al. The impact of mutations in SARS-CoV-2 spike on viral infectivity and antigenicity. Cell 182, 1284-1294.e9 (2020).

Publisher's note Springer Nature remains neutral with regard to jurisdictional claims in published maps and institutional affiliations.

(c) The Author(s), under exclusive licence to Springer Nature Limited 2021 


\section{Methods}

No statistical methods were used to predetermine sample size. The experiments were not randomized, and investigators were not blinded to allocation during experiments and outcome assessment except where indicated.

\section{Cell and culture conditions}

Vero E6 cells (a gift from M, Müller) were cultured in Dulbecco's modified Eagle's medium (DMEM) supplemented with $10 \%$ fetal bovine serum, $1 \times$ non-essential amino acids, 100 units $\mathrm{ml}^{-1}$ penicillin and $100 \mu \mathrm{g} \mathrm{ml}^{-1}$ streptomycin. Baby hamster kidney (BHK) cells expressing SARS-CoV nucleoprotein $(\mathrm{N})^{23,24}$ were maintained in minimal essential medium (MEM) supplemented with $5 \%$ fetal bovine serum (FBS), $1 \times$ non-essential amino acids, 100 units $\mathrm{ml}^{-1}$ penicillin and $100 \mu \mathrm{g} \mathrm{ml}^{-1}$ streptomycin, $500 \mu \mathrm{g} \mathrm{ml}^{-1} \mathrm{G} 418$ and $10 \mu \mathrm{g} \mathrm{ml}^{-1}$ puromycin. Twenty-four hours before electroporation, the BHK cells expressing SARS-CoV $\mathrm{N}$ were treated with $1 \mu \mathrm{g} \mathrm{ml}^{-1}$ doxycyclin to express SARS-CoV N protein. All cell lines were maintained at $37^{\circ} \mathrm{C}$ and in a $5 \% \mathrm{CO}_{2}$ atmosphere.

\section{Recombinant proteins}

The Expi293 Expression system (ThermoFisher Scientific) was used to produce hACE2 and S. Mammalian expression plasmids were constructed to encode codon-optimized Fc(human IgG1)-tagged hACE2 (hACE2-Fc) or polyhistidine-tagged S (S and S(D614G)), which contain the full-length ectodomain of S (residues 1-1208, with a mutated furin cleavage site and K986P/V987P substitutions). Expi293F cells were transfected with the plasmids and cultured at $37^{\circ} \mathrm{C}$ with $8 \% \mathrm{CO}_{2}$ at a shaking speed of $125 \mathrm{rpm}$. The supernatant was collected on day 5 by centrifugation at $3,000 \mathrm{~g}$ for $20 \mathrm{~min}$ at $4{ }^{\circ} \mathrm{C}$. The hACE2-Fc protein was purified using HiTrap Protein A column (GE Life Sciences) in elution buffer containing $0.1 \mathrm{M}$ citric acid, $\mathrm{pH}$ 3.0.S was purified using His Trap FF column (GE Life Sciences) in elution buffer containing $20 \mathrm{mM}$ sodium phosphate, $0.5 \mathrm{M} \mathrm{NaCl}$ and $500 \mathrm{mM}$ imidazole, $\mathrm{pH} 7.4$. The eluents were desalted using Zeba spin desalting column 7K MWCO (Thermo Fisher Scientific). The purified proteins were further concentrated on Amicon Ultra Centrifugal Filters 50K NMWL (Sigma-Aldrich) and quantified using Qubit protein assay (ThermoFisher Scientific).

\section{Biolayer interferometry assay}

Affinity between hACE2-Fc and S1 (ABclonal, RP01262), S1(D614G) (ABclonal, RP01287), S or S(D614G) was evaluated using Octet RED96 instrument (ForteBio) at $30^{\circ} \mathrm{C}$ with a shaking speed of $1,000 \mathrm{rpm}$. Following 20 min of prehydration of anti-human Fc capture biosensors and 1 min of sensor check, $7.5 \mathrm{nM} \mathrm{hACE} 2-\mathrm{Fc}$ in $10 \times$ kinetic buffer (ForteBio) was loaded onto the surface of biosensors for $5 \mathrm{~min}$. After $1.5 \mathrm{~min}$ of baseline equilibration, $5 \mathrm{~min}$ of association was conducted with 25 to $200 \mathrm{nM}$ of S1 or S1(D614G), or with 10-100 nM of S or S(D614G), followed by $5 \mathrm{~min}$ of dissociation in the same buffer used for baseline equilibration. The data were corrected by subtracting the reference sample; a 1:1 binding model with global fit was used for the determination of affinity constants.

\section{Flow cytometry}

A stable clone of BHK-hACE2 cells was pelleted and resuspended in reaction buffer ( $\mathrm{PBS} \mathrm{pH} 7.4$ with $0.02 \%$ Tween-20 and $4 \% \mathrm{BSA}$ ) at a concentration of $5 \times 10^{6}$ cells per $\mathrm{ml}$. One hundred microlitres per well of the cells were aliquoted into a round-bottom 96-well plate and incubated on ice for at least $5 \mathrm{~min}$. Polyhistidine-tagged S1 (40591-V08H) and S1(D614G) (40591-V08H3) and Fc-tagged S1 (40591-v02H) and S1(D614G) (40591-v02H3) proteins were purchased from Sino Biological, and diluted in reaction buffer on ice. Fifty microlitres of S1 diluents were added into corresponding wells of cells and incubated on ice for $20 \mathrm{~min}$ with shaking. After incubation, cells were washed in $200 \mu \mathrm{l}$ PBST washing solution (PBS pH7.4 with $0.02 \%$ Tween-20) once, and then $100 \mu \mathrm{l}$ of 1:300 diluted secondary antibody (ThermoFisher cat. no. A-21091 for the Fc tag and ThermoFisher cat. no. MA1-21315-647 for the polyhistidine tag) was added into each well of cells, mixed and incubated on ice with shaking for $15 \mathrm{~min}$. After washing twice, cells were resuspended in $200 \mu \mathrm{l}$ PBST and analysed using a BD FACSCanto II Flow Cytometer. Data were processed using Flowjo v.10.6.1. Results for BHK control cells (which do not express hACE2) and the gating strategy are provided in Supplementary Figs. 1, 2.

\section{Generation of infectious cDNA clones using transformation-associated recombination cloning and rescue of recombinant viruses}

To introduce the mutation for the D614G substitution into the $S$ gene, PCR mutagenesis (Supplementary Table1) was performed on the pUC57 plasmid containing SARS-CoV-2 fragment $10^{10}$ using a Q5 Site-Directed Mutagenesis Kit (New England BioLab). Both $S$ and $S^{D 614 G}$ infectious cDNA clones were generated using in-yeast transformation-associated recombination cloning method as previously described ${ }^{10}$. In vitro transcription was performed for Eagl-cleaved YAC and PCR-amplified SARS-CoV-2 $N$ gene using the T7 RiboMAX Large Scale RNA production system (Promega) as previously described ${ }^{4}$. Transcribed capped mRNA was electroporated into baby hamster kidney cells (BHK-21 cells) expressing SARS-CoV N protein. Electroporated cells were cocultured with susceptible Vero E6 cells to produce passage 0 of recombinant SARS-CoV-2 ${ }^{\mathrm{D} 614}$ and SARS-CoV-2 ${ }^{\mathrm{G} 614}$. Subsequently, progeny viruses were used to infect fresh Vero $\mathrm{E} 6$ cells to generate passage 1 stocks for downstream experiments.

\section{Virus growth kinetics and plaque assay}

The characterization of virus growth kinetics in Vero E6 was performed as previously described ${ }^{10}$. Twenty-four hours before infection, cells were seeded in a 24 -well plate at a density of $2.0 \times 10^{5}$ cells per ml. After washing once with PBS, cells were inoculated with viruses at multiplicity of infection of 0.01 . After $1 \mathrm{~h}$, the inoculum was removed and cells were washed three times with PBS followed by supply with appropriate culture medium.

PFUs per ml of recombinant SARS-CoV-2 ${ }^{\mathrm{D} 614}$ and SARS-CoV-2 ${ }^{\mathrm{G} 614}$ were determined by plaque assay in a 24-well format. One day before infection, Vero E6 cells were seeded at a density of $2.0 \times 10^{5}$ cells per $\mathrm{ml}$. After washing once with PBS, cells were inoculated with viruses serially diluted in cell culture medium at 1:10 dilution. After $1 \mathrm{~h}$ of incubation, inoculum was removed, and cells were washed with PBS and subsequently overlaid with $1: 1$ mix of $2.4 \%$ Avicel and $2 \times$ DMEM supplemented with $20 \%$ fetal bovine serum, 200 units $\mathrm{ml}^{-1}$ penicillin and $200 \mathrm{\mu g} \mathrm{ml}^{-1}$ streptomycin. After 2 days of incubation at $37^{\circ} \mathrm{C}$, cells were fixed in $4 \%(v / v)$ neutral-buffered formalin before stained with crystal violet.

Statistical significance was determined by two-sided unpaired Student's $t$-test without adjustment for multiple comparisons.

\section{Infection of human nasal and bronchial epithelial cells}

Primary human nasal epithelial cell cultures (MucilAir EP02, Epithelix Sàrl) were purchased and handled according to the manufacturer's instructions. Human NBE cells were purchased (from Emory University) and cultured according to recommended protocols. The human nasal epithelial cultures were inoculated with $0.5 \times 10^{5} \mathrm{PFU}$ per well, or mixtures of 1:1, 10:1 and 1:10 of SARS-CoV-2 ${ }^{\mathrm{D} 614}$ and SARS-CoV-2 ${ }^{\mathrm{G} 614}$. Human NBE cell cultures were inoculated with $1.0 \times 10^{5} \mathrm{PFU}$ per well, or with the wild-type isolates SARS-CoV-2/USA-WA1/2020 (SARS-CoV-2 ${ }^{\text {D614 }}$ ) or SARS-CoV-2/Massachusetts/VPT1/2020 (SARS-CoV-2 ${ }^{\mathrm{G} 614}$ ), at $2 \times 10^{5}$ $\mathrm{TCID}_{50}$ per well. For competition experiments, human NBE cells were inoculated with 1:1 or 9:1 mixed SARS-CoV-2 $2^{\mathrm{D} 614}$ and SARS-CoV- ${ }^{\mathrm{G} 614}$ at $1 \times 10^{5} \mathrm{PFU}$ per well. After incubation at $33^{\circ} \mathrm{C}$ for 1 or $2 \mathrm{~h}$, for human nasal epithelial or human NBE cell cultures, respectively, inoculum was removed (or collected for human NBE as 2-h samples), cells were washed, and subsequently incubated (as indicated) at $33^{\circ} \mathrm{C}, 37^{\circ} \mathrm{C}$ or 
$39^{\circ} \mathrm{C}$. To monitor viral replication, apical washes were collected every $24 \mathrm{~h}$. All titre were determined by standard plaque assay or $\mathrm{TCID}_{50}$ on Vero E6 cells.

For competition experiments, viral RNA was extracted from apical washes using the QIAamp 96 Virus QIAcube HT Kit (QIAGEN). The SARS-CoV-2 genome was amplified using a highly multiplexed tiling PCR reaction based on the Artic Network protocol (https://www.protocols.io/view/ncov-2019-sequencing-protocol-bbmuik6w) with some modification. In brief, primers were designed to produce overlapping 1-kb amplicons (Supplementary Table 1). Reverse transcriptase was performed as described in the Artic Network protocol. The single cDNA reaction was carried forward by preparing two PCR reactions (one each for the odd and even pool of primers). Two primer pools (odd and even) were prepared to contain $0.1 \mu \mathrm{M}$ of each individual primer. Tiling PCR of the resultant cDNA was performed by combining $12.5 \mu \mathrm{l}$ $2 \times \mathrm{Q} 5$ polymerase, $5.5 \mu \mathrm{l}$ water, $2 \mu \mathrm{l}$ of the primer pool and $5 \mu \mathrm{l}$ of cDNA followed by incubating the reaction at $98^{\circ} \mathrm{C}$ for $30 \mathrm{~s}, 38$ cycles of $98^{\circ} \mathrm{C}$ for $15 \mathrm{~s}$ and $63^{\circ} \mathrm{C}$ for $5 \mathrm{~min}$, and holding at $4^{\circ} \mathrm{C}$. Corresponding odd and even amplicons were normalized and pooled for library preparation. Fragmented libraries were prepared using the Nextera XT DNA library preparation kit and sequenced using Illumina MiSeq. Analyses were performed using IRMA ${ }^{25}$ with a SARS-CoV-2 module.

\section{RNA extraction and RT-PCR}

Preparation of viral RNA for NGS was performed as previously described $^{10}$. In brief, Vero E6 cells were infected with passage-1 viruses. Extraction of total cellular RNA was done using Nucleospin RNA Plus kit (Macherey-Nagel) according to the manufacturers' instructions.

RNA from apical washes of human nasal epithelial cells and mouse oropharyngeal swabs were prepared using QIAamp Viral RNA Mini Kit (QIAGEN) and Nucleospin RNA kit (Macherey-Nagel) according to the manufacturers' protocols.

To determine the ratios of SARS-CoV-2 ${ }^{\mathrm{D} 614}$ to SARS-CoV-2 ${ }^{\mathrm{G} 614}$ in competition assays in human nasal epithelial cell cultures and hACE2 knock-in mice, reverse-transcription PCR was performed on extracted RNA using SuperScript IV One-step RT-PCR System (Invitrogen). The sequence-specific primers were used to generate an amplicon of 905 bp covering the D614 region: 5'-AATCTATCAGGCCGGTAGCAC- ${ }^{\prime}$ ' and 5'-CAACAGCTATTCCAGTTAAAGCAC-3'. RT-PCR reaction was performed in a $50-\mu \mathrm{l}$ reaction using $0.01 \mathrm{pg}$ to $1 \mu \mathrm{g}$ total RNA. The cycling parameters were set as follows: $50^{\circ} \mathrm{C}$ for $10 \mathrm{~min}, 98^{\circ} \mathrm{C}$ for $2 \mathrm{~min} ; 35$ cycles at $98^{\circ} \mathrm{C}$ for $10 \mathrm{~s}, 55^{\circ} \mathrm{C}$ for $15 \mathrm{~s}$, and $72^{\circ} \mathrm{C}$ for $30 \mathrm{~s}$; and a 5 -min incubation at $72^{\circ} \mathrm{C}$. DNA concentration was determined using Qubit dsDNA HS (High Sensitivity) Assay (Thermo Fisher), and subsequently diluted to $200 \mathrm{ng}$ in $50 \mu \mathrm{l}$ of nuclease-free water for sequencing by Nanopore sequencing MinION.

RNA extraction and preparation of RT-PCR reactions were performed in low-and no-copy laboratory environment to avoid contamination.

\section{Sequencing and computational analysis}

Recombinant SARS-CoV-2 ${ }^{\mathrm{D} 614}$ or SARS-CoV-2 ${ }^{\mathrm{G} 614}$ RNAs of passage-1 stock were sequenced by NGS as previously described ${ }^{10}$. In brief, RNA was extracted from Vero E6 cells infected with either recombinant SARS-CoV-2 ${ }^{\mathrm{D} 614}$ or SARS-CoV-2 ${ }^{\mathrm{G} 614}$ using the NucleoSpin RNA Plus kit (Macherey-Nagel) according to the manufacturer's guidelines. Sequencing libraries were subsequently prepared using the Illumina TruSeq Stranded mRNA Library Prep Kit (Illumina, 20020595) and pooled cDNA libraries were sequenced across two lanes on a NovaSeq 6000 S1 flow cell $(2 \times 50$ bp, 100 cycles $)$ using the Illumina NovaSeq 6000 platform (Next Generation Sequencing Platform of the University of Bern). For data analysis, TrimGalore v.0.6.5 was used to remove low-quality reads and adaptors from the raw sequencing files. The resulting trimmed paired-end reads were then aligned to the SARS-CoV-2 genome (GenBank accession MT108784) using Bowtie2 v.2.3.5. Finally, a consensus sequence was generated for each virus stock using Samtools v.1.10 with the - $d$ option set to 10,000. Data analysis was performed on UBELIX, the high-performance computing cluster at the University of Bern (http://www.id.unibe.ch/hpc).

For virus competition experiments in human nasal epithelial cells, hACE2 knock-in mice, hamsters and ferrets, amplicons were sequenced on the MinION system from Oxford Nanopore. Real-time high-accuracy base calling, demultiplexing and barcode and adaptor trimming was performed with MinKnow v.20.06.17, running Guppy v.s4.0.11. Downstream analysis was done in Geneious 2019 v.s2.3. Read length was filtered to eliminate reads $<800$ and $>1,100 \mathrm{nt}$ and the remaining reads were mapped in subsets of 10,000 reads to the amplicon reference undergoing 2 iterations, with custom sensitivity allowing a maximum of $5 \%$ gaps and maximum mismatch of $30 \%$. Variants were analysed at specific positions calculating $P$ values for each variant. The ratio fraction $\mathrm{A} / \mathrm{G}$ was calculated from the numbers of reads, as fraction $=\mathrm{A}$ reads/(A reads $+G$ reads).

Statistical analyses for virus competition experiments and relative replicative fitness calculations were performed using the catseyes package in R. Relative replicative fitness values for SARS-CoV- ${ }^{\mathrm{G} 614}$ over SARS-CoV-2 ${ }^{\mathrm{D} 614}$ were determined by dividing the initial SARS-CoV-2 ${ }^{\mathrm{G} 614}$ / SARS-CoV- $2^{\mathrm{D} 614}$ ratio $\left(i_{0}\right)$ by the final (after infection) SARS-CoV- $2^{\mathrm{G} 614} /$ SARS-CoV-2 ${ }^{\mathrm{D} 614}$ ratio $\left(f_{0}\right)$ according to the formula $w=\left(f_{0} / i_{0}\right)$. Specifically, to model $f_{0} / i_{0}$ in each experiment, the $t_{1} / t_{0}$ ratio was calculated on the basis of the sequencing counts attained for each virus in individual samples (after infection, time point $t_{1}$ ) and in the inoculum (initial time point $t_{0}$ ). For each time point, a linear regression model was generated in $\mathrm{R}$ and fitness ratios between the two groups (variants) were assessed by means of the coefficient of the model's group term. When multiple experiments were performed, the experiment variable was included in the model. All statistical tests were performed in R (v.4.0.2) with two-sided $\alpha=0.05$. Cat's eye plots showing the relative replicative fitness of SARS-CoV-2 ${ }^{\mathrm{G} 614}$ over SARS-CoV-2 ${ }^{\mathrm{D} 614}$ for each time point in each experiment were created using the catseyes package in $\mathrm{R}^{26}$, as previously described $^{20}$.

\section{Animal studies}

Ethics declarations. The hACE2 knock-in mice were originally generated at the Wadsworth Center, New York State Department of Health (IACUC protocol no. 09-405) (principal investigator D.E.W.). Mouse experiments were conducted in compliance with the Swiss Animal Welfare legislation, and animal studies were reviewed and approved by the commission for animal experiments of the canton of Bern under licence $\mathrm{BE}-43 / 20$. All of the ferret and hamster experiments were evaluated by the responsible ethics committee of the State Office of Agriculture, Food Safety, and Fishery in Mecklenburg-Western Pomerania (LALLF $\mathrm{M}-\mathrm{V})$, and gained governmental approval under registration number LVL MV TSD/7221.3-1-041/20. This project also obtained clearance from the Animal Care and Use Program Office of the CDC.

hACE2 knock-in mouse study. For the generation of hACE2 knock-in mice, the hACE2 knock-in (B6) (B6.Cg-ACe $2^{\text {tm1(ACE2)Dwnt } / J) ~ l i n e ~ w a s ~ g e n e r-~}$ ated by targeted mutagenesis to express human $A C E 2$ CDNA in place of the mouse $A c e 2$ gene. Thus, in this mouse model, hACE2 expression is regulated by the endogenous mouse Ace 2 promoter and enhancer elements. The targeting vector (WEN1-HR) had human ACE2 cDNA inserted in frame with the endogenous initiation codon of the mouse Ace2 (Extended Data Fig. 3a). The human cDNA was flanked by an FRT-neomycin-FRT-loxP cassette and a distal loxP site. WEN1-HR was used to transfect 129Sv/Pas embryonic stem cells and $837 \mathrm{em}$ bryonic stem cell clones were isolated and screened for homologous recombination by PCR and Southern blot. Eleven properly recombined embryonic stem cell clones were identified, and some of them were used for blastocyst injection and implantation into female mice to generate 22 male founder mice with chimerism (129Sv/Pas:C57BL/6) ranging from 50 to $100 \%$. To complete the hACE2 knock-in, we crossed 
the chimeric male mice with $\mathrm{C} 57 \mathrm{BL} / 6 \mathrm{~J}$ Flp-expressing female mice to excise the FRT-flanked neomycin selection cassette and generate the floxed humanized $A C E 2$ allele (Extended Data Fig. 3a). These hACE2 knock-in mice were identified by PCR and confirmed by Southern blot and were backcrossed to $\mathrm{C} 57 \mathrm{BL} / 6 \mathrm{~J}$ mice for seven generations before this study. This line has been donated to The Jackson Laboratory for use by the scientific community (stock 035000).

Heterozygous hACE2 knock-in female mice were obtained from The Jackson Laboratory and C57BL/6J wild-type female mice were from Janvier Lab. All mice were acclimatized for at least 2 weeks in individually ventilated cages (blue line, Tecniplast), with 12-h/12-h light/dark cycle, $22 \pm 1^{\circ} \mathrm{C}$ ambient temperature and $50 \pm 5 \%$ humidity, autoclaved acidified water, autoclaved cages including food, bedding, and environmental enrichment at the specific-pathogen-free facility of the Institute of Virology and Immunology, (Mittelhäusern). One week before infection, mice were placed in individually HEPA-filtered isolators (IsoCage N, Tecniplast). Mice (10 to 12 weeks old) were anaesthetized with isoflurane and infected intranasally with $20 \mu \mathrm{l}$ (that is, $10 \mu$ l per nostril) with a 1:1 ratio of the SARS-CoV-2 variants (wild-type and hACE2 knock-in mice) or mock culture medium (wild-type mice only). After intranasal infection, mice were monitored daily for body weight loss and clinical signs. Throat swabs were collected daily under brief isoflurane anaesthesia using ultrafine sterile flock swabs (Hydraflock, Puritan, 25-3318-H). The tips of the swabs were placed in $0.5 \mathrm{ml}$ of RA1 lysis buffer (Macherey-Nagel, ref. 740961) supplemented with $1 \% \beta$-mercaptoethanol and vortexed. Groups of mice from two independent experiments were euthanized on days 2 and 4 after infection and organs were aseptically dissected, avoiding cross-contamination. Systematic tissue sampling was performed: (1) lung right superior lobe, right nasal concha, right olfactory bulb, part of the right brain hemisphere, apical part of the right kidney and parts of the distal small intestine (ileum) were collected for RNA isolation in RA1 lysis buffer; (2) lung middle, inferior and post-caval lobes, left nasal concha, left olfactory bulb, part of the right brain hemisphere and part of the right kidney were collected in MEM; and (3) lung left lobe, liver left lobe, left kidney, left brain hemisphere and part of the jejunum and ileum were fixed in buffered formalin. Data were generated from two identically designed independent experiments.

Mouse tissue samples collected in RA1 lysis buffer supplemented with $1 \% \beta$-mercaptoethanol were homogenized using a Bullet Blender Tissue Homogenizer (Next-Advance). Homogenates were centrifuged for $3 \mathrm{~min}$ at $18,000 \mathrm{~g}$ and stored at $-70^{\circ} \mathrm{C}$ until processing. Total RNA was extracted from homogenates using the NucleoMag VET kit for viral and bacterial RNA and DNA from veterinary samples (Macherey Nagel, ref. 744200) and the KingFisher Flex automated extraction instrument (ThermoFisher Scientific) following the manufacturers' instructions. RNA purity was analysed with a NanoDrop 2000 (ThermoFisher Scientific). A $25-\mu \mathrm{l}$ RT-PCR for the viral $E$ gene was carried out using $5 \mu \mathrm{l}$ of extracted RNA template using the AgPath-ID One-Step RT-PCR (Applied Biosystems). Samples were processed in duplicate. Amplification and detection were performed in an Applied Biosystem 7500 Real-Time PCR System under the following conditions: an initial reverse transcription at $45^{\circ} \mathrm{C}$ for $10 \mathrm{~min}$, followed by PCR activation at $95^{\circ} \mathrm{C}$ for $10 \mathrm{~min}$ and 45 cycles of amplification $\left(15 \mathrm{~s}\right.$ at $95^{\circ} \mathrm{C}, 30$ s at $56^{\circ} \mathrm{C}$ and $30 \mathrm{~s}$ at $\left.72^{\circ} \mathrm{C}\right)$.

Fixed mouse tissue samples were processed, sectioned and stained with haematoxylin and eosin at the COMPATH core facility (University of Bern). Histopathological lung slides of hACE2 knock-in mice and wild-type mice (infected and mock) were examined and scored by a board-certified veterinary pathologist (S.d.B.), who was blinded to the identity of the samples. Scoring criteria are detailed in Supplementary Table 4.

Hamster study. Six Syrian hamsters (Mesocricretus auratus) (Janvier Labs) were infected intranasally under a short-term inhalation anaesthesia with $70 \mu$ of SARS-CoV-2 ${ }^{\mathrm{D} 614}$ and SARS-CoV-2 ${ }^{\mathrm{G} 614}$ at equal ratios using $10^{4.77} \mathrm{TCID}_{50}$ per hamster (calculated from back titration of the original material). After $24 \mathrm{~h}$ of isolated housing in individually ventilated cages, six pairs (each with one directly inoculated donor hamster and one sham-inoculated contact hamster) were cohoused. The housing of each hamster pair was strictly separated in individual cage systems to prevent spillover between different pairs. For the following seven days (day 2 until day 8 after infection) and on day 12 after infection, viral shedding was monitored in addition to a daily physical examination and body weighing routine.

To evaluate viral shedding, nasal washes were individually collected from each hamster under a short-term isoflurane anaesthesia. Starting with the contact hamster of each pair, each nostril was rinsed with $100 \mu \mathrm{l}$ PBS (1.0× phosphate-buffered saline) and reflux was immediately gathered. A new pipette tip for every nostril and hamster was used to prevent indirect spillover transmission from one hamster to another. Furthermore, in between the respective pairs, a new anaesthesia system was used for each pair of hamsters. At day 8 after infection, one contact hamster was found dead. Although they lost up to almost $20 \%$ of their body weight, every other hamster recovered from disease. Under euthanasia, serum samples were collected from each hamster.

For the single-virus infection experimental setup, seven hamsters each were infected via the intranasal route with $10^{5.1} \mathrm{TCID}_{50}$ per hamster of SARS-CoV-2 ${ }^{\mathrm{D} 614}$, or $10^{4.5} \mathrm{TCID}_{50}$ per hamster of SARS-CoV-2 ${ }^{\mathrm{G} 614}$ (calculated from back titration of the original material). From day 1 to day 4, nasal washes were obtained from these hamsters and body weight changes recorded. A tissue panel from respiratory organs-including nasal conchae, tracheal tissue, cranial, medial and caudal portions of the right lung, and the pulmonary lymph node-were collected after euthanasia from these hamsters.

Ferret study. In accordance with the hamster study, twelve ferrets (Mustela putoriusfuro) from in-house breeding were divided into six groups of two individuals. Ferret pairs were of equal age (between 4 and 18 months). In total, four ferrets were female (two pairs) and eight ferrets were male or neutered male (four pairs). The housing of each ferret pair was strictly separated in individual cage systems, to prevent spillover between different pairs. Per separate cage, one individual ferret was inoculated intranasally by instillation of $125 \mu \mathrm{l}$ of the aforementioned inoculum $\left(10^{5.4} \mathrm{TCID}_{50}\right.$ per ferret, calculated from back titration of the equally mixed original material) into each nostril under a short-term isoflurane-based inhalation anaesthesia. Time points and sampling technique corresponded to the methods used for the hamsters, although ferret nasal washing was performed using $2 \times 700 \mu \mathrm{l}$ PBS per ferret. The contact ferret was not inoculated.

Specimen work up, viral RNA detection, and sequencing and quantification analyses. Organ samples were homogenized in a $1 \mathrm{ml} \mathrm{mix-}$ ture of equal volumes composed of Hank's balanced salts MEM and

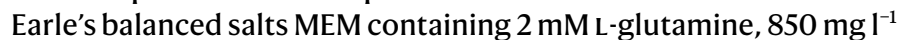
$\mathrm{NaHCO}_{3}, 120 \mathrm{mg} \mathrm{l}^{-1}$ sodium pyruvate supplemented with $10 \%$ fetal calf serum and $1 \%$ penicillin-streptomycin at $300 \mathrm{~Hz}$ for $2 \mathrm{~min}$ using a Tissuelyser II (Qiagen) and centrifuged to clarify the supernatant. Nucleic acid was extracted from $100 \mu$ l of the nasal washes after a short centrifugation step or organ sample supernatant using the NucleoMag Vet kit (Macherey Nagel). Viral loads in these samples were determined using the nCoV_IP4 RT-PCR ${ }^{27}$ including standard RNA that were absolute-quantified by digital droplet PCR with a sensitivity of 10 copies per reaction. The extracted RNA was afterwards subjected to MinION sequencing and digital droplet PCR. For MinION sequencing, amplicons were produced with primers (WU-21-F: AATCTATCAGGCCG GTAGCAC; WU-86-R: CAACAGCTATTCCAGTTAAAGCAC) using SuperScript IV One-step RT-PCR (Thermo Fisher Scientific). Amplicons were sequenced on a MinION system from Oxford Nanopore using Native Barcoding 1-12 (EXP-NBD104) and 13-24 (EXP-NBD114), respectively, with Ligation Kit SQK-LSK109 (Oxford Nanopore). Libraries were loaded 
on R9.4.1 Flow Cells (FLO-MIN106D) on a MinION coupled to a MinIT. MinION data analysis was performed as described in 'Sequencing and computational analysis'.

For rare mutation and sequence analysis based on digital droplet PCR, the QX200 Droplet Digital PCR System from Bio-Rad was used. The One-Step RT-ddPCR Advanced Kit for Probes (Bio-Rad) was applied according to the supplier's instructions. For the amplification of an 86-bp fragment of both variants of $S$, the forward primer SARS2-S-1804-F (5'-ACA AAT ACT TCT AAC CAG GTT GC-3') and the reverse primer SARS2-S-1889-R (5'-GTA AGT TGA TCT GCA TGA ATA GC- $3^{\prime}$ ) were used. For the detection of the $D$ and $G$ variants in one sample, two allele-specific locked nucleic acid probes were applied: SARS2-S-v1D-1834FAM (5'-FAM-TaT cAG gat GTt AAC-BHQ1-3') and SARS2-S-v3G-1834HEX (5'-HEX-T cAG ggt GTt AAC-BHQ1-3') (the locked nucleic acid positions are in lowercase). The concentration of the primers and probes was $20 \mu \mathrm{M}$ and $5 \mu \mathrm{M}$, respectively. For data analysis, the QuantaSoft Analysis Pro software (v.1.0.596) was used.

\section{Reporting summary}

Further information on research design is available in the Nature Research Reporting Summary linked to this paper.

\section{Data availability}

Sequence data are available on the NCBI Sequence Read Archive (SRA) under the BioProject accession number PRJNA669553. Source data are provided with this paper.

23. van den Worm, S. H. et al. Reverse genetics of SARS-related coronavirus using vaccinia virus-based recombination. PLoS ONE 7, e32857 (2012)

24. Thiel, V., Herold, J., Schelle, B. \& Siddell, S. G. Infectious RNA transcribed in vitro from a cDNA copy of the human coronavirus genome cloned in vaccinia virus. J. Gen. Virol. 82, 1273-1281 (2001).

25. Shepard, S. S. et al. Viral deep sequencing needs an adaptive approach: IRMA, the iterative refinement meta-assembler. BMC Genomics 17, 708 (2016).
26. Anderson, C. Catseyes: create catseye plots illustrating the normal distribution of the means. R package version 0.2.3. https://CRAN.R-project.org/package=catseyes (2019)

27. WHO. Protocol: real-time RT-PCR assays for the detection of SARS-CoV-2, https://www. who.int/docs/default-source/coronaviruse/

real-time-rt-pcr-assays-for-the-detection-of-sars-cov-2-institut-pasteur-paris. pdf?sfvrsn=3662fcb6_2 (accessed 3 December 2020).

Acknowledgements This work was supported by the Swiss National Science Foundation (SNF) (grants 31CA30 196644, 31CA30 196062 and 310030 173085), the European Commission (Marie Skłodowska-Curie Innovative Training Network 'HONOURS'; grant agreement no. 721367, and the Horizon 2020 project 'VEO' grant agreement no. 874735), the Federal Ministry of Education and Research (BMBF) (grant RAPID, no. 01KI1723A) and by core funds of the University of Bern and the German Federal Ministry of Food and Agriculture. The hACE2 knock-in (B6) mice were generated with support from NIH/NIAID P01AI059576 (subproject 5) and partial support from NIH/NIAID U54-AI-057158. Partial support was provided by the US Centers for Disease Control and Prevention COVID-19 Task Force. This work was supported by COVID-19 special funds from the Swiss Federal Office of Public Health and, from the Swiss Federal Office of Food Safety and Veterinary Affairs, and we thank A. Summerfield for help in securing Swiss government funds. We thank the NGS platform of the University of Bern; M. Lange, C. Korthase, M. Currie, G. Larson and S. Renzullo for their excellent technical assistance; and F. Klipp, D. Fiedler, H. Manthei, C. Lipinski, J. Tang and A. Godel for their invaluable support in the animal experiments. This activity was reviewed by the CDC and was conducted in a manner consistent with applicable federal laws and CDC policies: 45 C.F.R. part 46, 21 C.F.R. part 56; 42 U.S.C. Sect. 241(d); 5 U.S.C. Sect. 552a; 44 U.S.C. Sect. 3501 et seq. The findings and conclusions in this Article are those of the authors and do not necessarily represent the official position of the US Centers for Disease Control and Prevention.

Author contributions V.T., D.E.W., M.B. and C.B. conceived the study. T.T.N.T., B.Z., D.H. and A.T performed most of the experiments. N.E., S.S., F.L., J.N.K., H.S., J.P., B.S.T., J.J., R.D., D.H., N.J.H L.U., J.K., B.P., A.P., B.H., X.F., X.L., L.W., N.J., D.C., J.H., M.M.W., M.W.K., T.J.S., J.R.B., S.d.B. and C.B. conducted experimental work and/or provided essential experimental systems, data analysis and reagents. V.T., D.E.W., M.B., C.B., T.T.N.T., B.Z., N.E., S.S., L.T., D.H., N.J.H. and L.U. wrote the manuscript and made the figures. All authors read and approved the final manuscript.

Competing interests The authors declare no competing interests.

Additional information

Supplementary information The online version contains supplementary material available at https://doi.org/10.1038/s41586-021-03361-1.

Correspondence and requests for materials should be addressed to C.B., D.E.W., V.T. or M.B. Peer review information Nature thanks Stanley Perlman and the other, anonymous, reviewer(s) for their contribution to the peer review of this work. Reprints and permissions information is available at http://www.nature.com/reprints. 


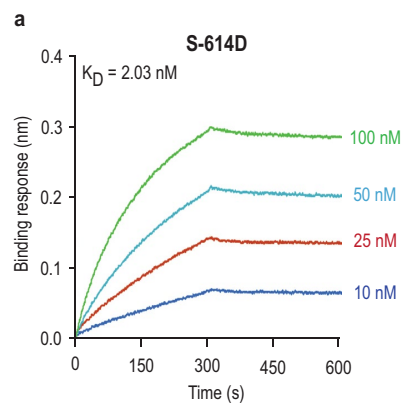

c

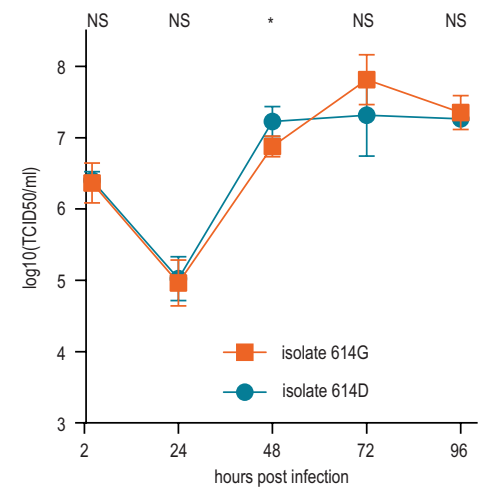

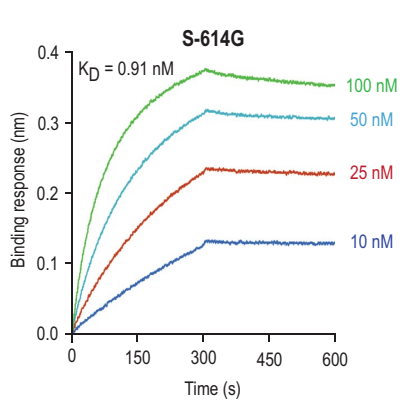

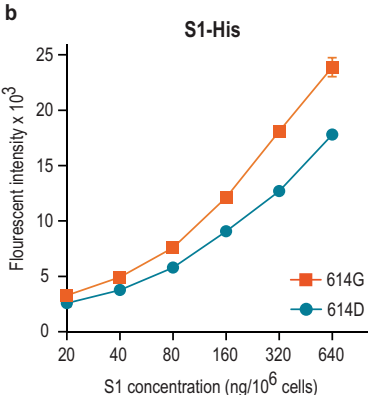

NhBE $37^{\circ} \mathrm{C}$

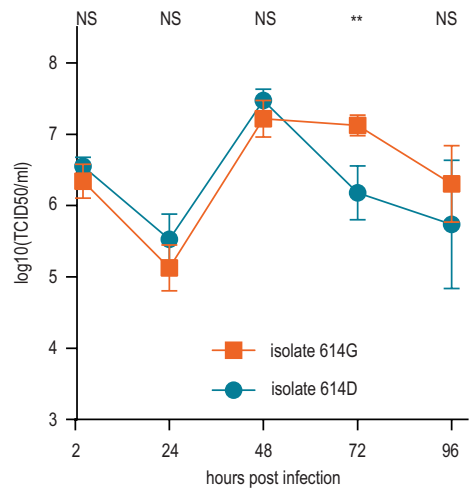

NhBE $39^{\circ} \mathrm{C}$

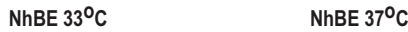

NS NS NS NS NS

NS * NS * *
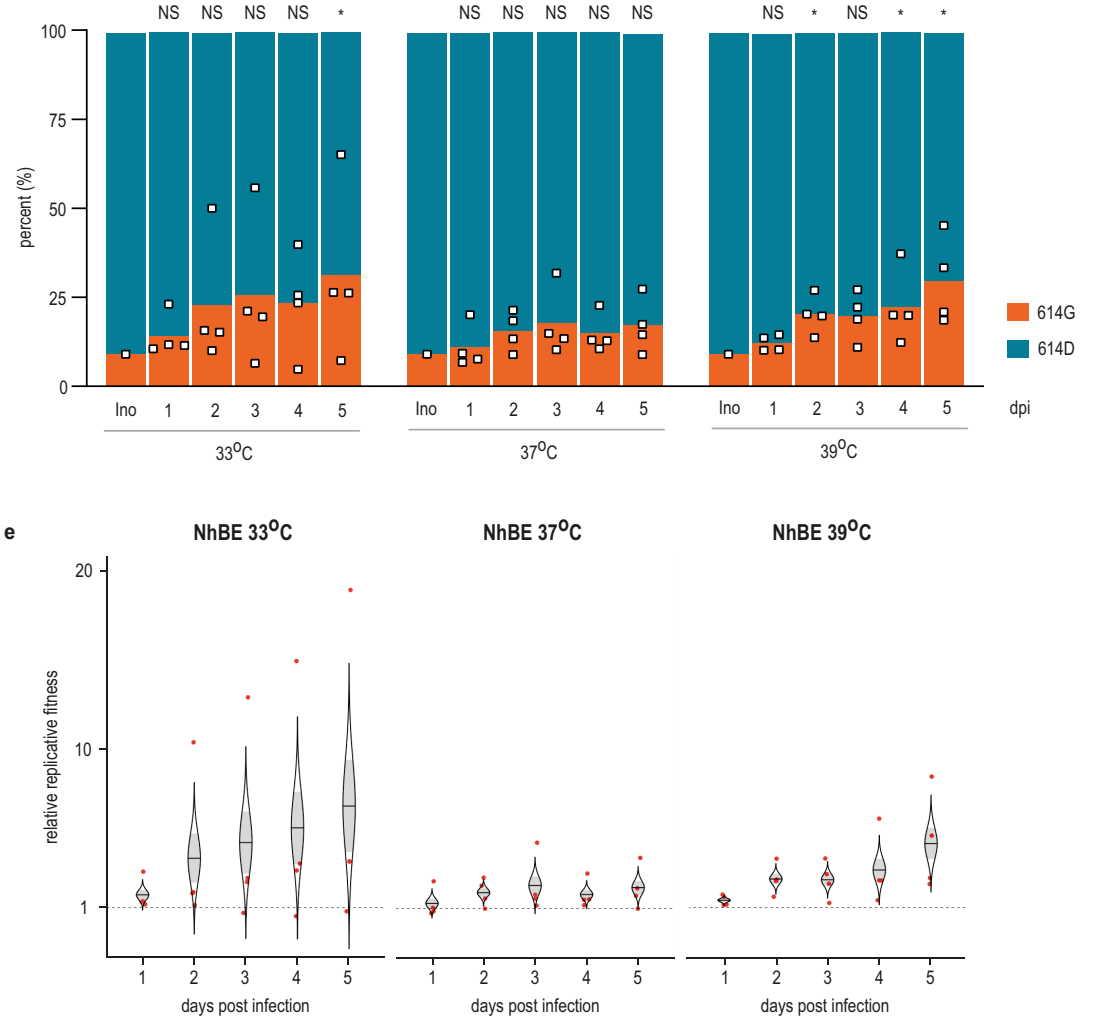

Extended Data Fig. 1 |See next page for caption. 
Extended Data Fig. 1 | Additional in vitro characterization of S and of SARSCoV-2 ${ }^{\mathrm{D} 614}$ and SARS-CoV-2 ${ }^{\mathbf{6} 614}$ isolates. a, Affinity between S and hACE2 determined by biolayer interferometry. Fc-tagged hACE2 was loaded onto surface of anti-human Fc capture biosensors and association was conducted using S (S-614D) or S(D614G) (S-614G) followed by dissociation. Data represent three biological replicates, and all repeats with statistical analysis are provided in Supplementary Table 2. b. Binding of polyhistidine-tagged or Fc-tagged S1 to BHK-hACE2 cells, determined by flow cytometry. Mean fluorescence intensity is shown for the corresponding S1 protein concentration. Data are mean \pm s.d. of three biological replicates. c, Replication of the isolates SARS-CoV-2/USAWA1/2020 (isolate 614D) and SARS-CoV-2/Massachusetts/VPT1/2020 (isolate 614G) in human NBE cells at $33^{\circ} \mathrm{C}$ (left), $37^{\circ} \mathrm{C}$ (middle) and $39^{\circ} \mathrm{C}$ (right). Human NBE cells were infected with $200,000 \mathrm{TCID}_{50}$ of each virus. Supernatants were collected every $24 \mathrm{~h}$ and titrated by $\mathrm{TCID}_{50}$ assay. Data are mean \pm s.d. of four replicates. Statistical significance was determined by two-sided unpaired Student's $t$-test without adjustments for multiple comparisons. $P$ values (from left to right): left, NS (not significant), $P=0.8098$; NS, $P=0.7874 ;{ }^{*} P=0.0328$; NS, $P=0.1887$; and NS, $P=0.5296$; middle, NS, $P=0.1689$; NS,$P=0.1475$; NS,
$P=0.1415 ;{ }^{* *} P=0.0033 ;$ and NS,$P=0.3184 ;$ right, ${ }^{*} P=0.0197 ; \mathrm{NS}, P=0.6018 ; \mathrm{NS}$, $P=0.3903$; NS, $P=0.0898$; and ${ }^{*} P=0.0445$. d, Competition assay of recombinant SARS-CoV-2 ${ }^{\mathrm{D} 614}$ (614D) and SARS-CoV-2 ${ }^{\mathrm{G} 614}$ (614G) in human NBE cells at $33^{\circ} \mathrm{C}, 37^{\circ} \mathrm{C}$ and $39^{\circ} \mathrm{C}$. The inoculum was prepared by mixing two viruses at 9:1 ratio based on PFU per ml. Human NBE cells were infected with 100,000 PFU of the 9:1 virus mix $(n=4)$. Viral RNA was extracted from daily supernatant and sequenced by NGS. Percentage of sequencing reads encoding S or S(D614G). Each square represents one individual data point in competition experiment. For each time point, a linear regression model was generated based on the sequence read counts for S and S(D614G), and $P$ values were calculated for the group (variant) coefficient. $P$ values (left to right): left, NS, $P=0.1796$; NS,$P=0.087$; NS,$P=0.1147$; NS,$P=0.1244$; and ${ }^{*} P=0.0401$; middle, $\mathrm{NS}, P=0.9114$; NS,$P=0.0715$; NS, $P=0.1696$; NS,$P=0.1696$; and NS,$P=0.1657$; right, NS, $P=0.1041 ; * P=0.013$; NS,$P=0.0645 ;{ }^{*} P=0.0102$; and ${ }^{*} P=0.0308$. e, Cat's eye plot illustrating the relative replicative fitness values of SARS-CoV$2^{\mathrm{G} 614}$ over SARS-CoV-2 ${ }^{\mathrm{D} 614}$ in human NBE cells for the competition experiments performed in d. Each dot represents an individual data point, the centre line represents the mean and the shaded area represents the s.d. 


\section{Article}
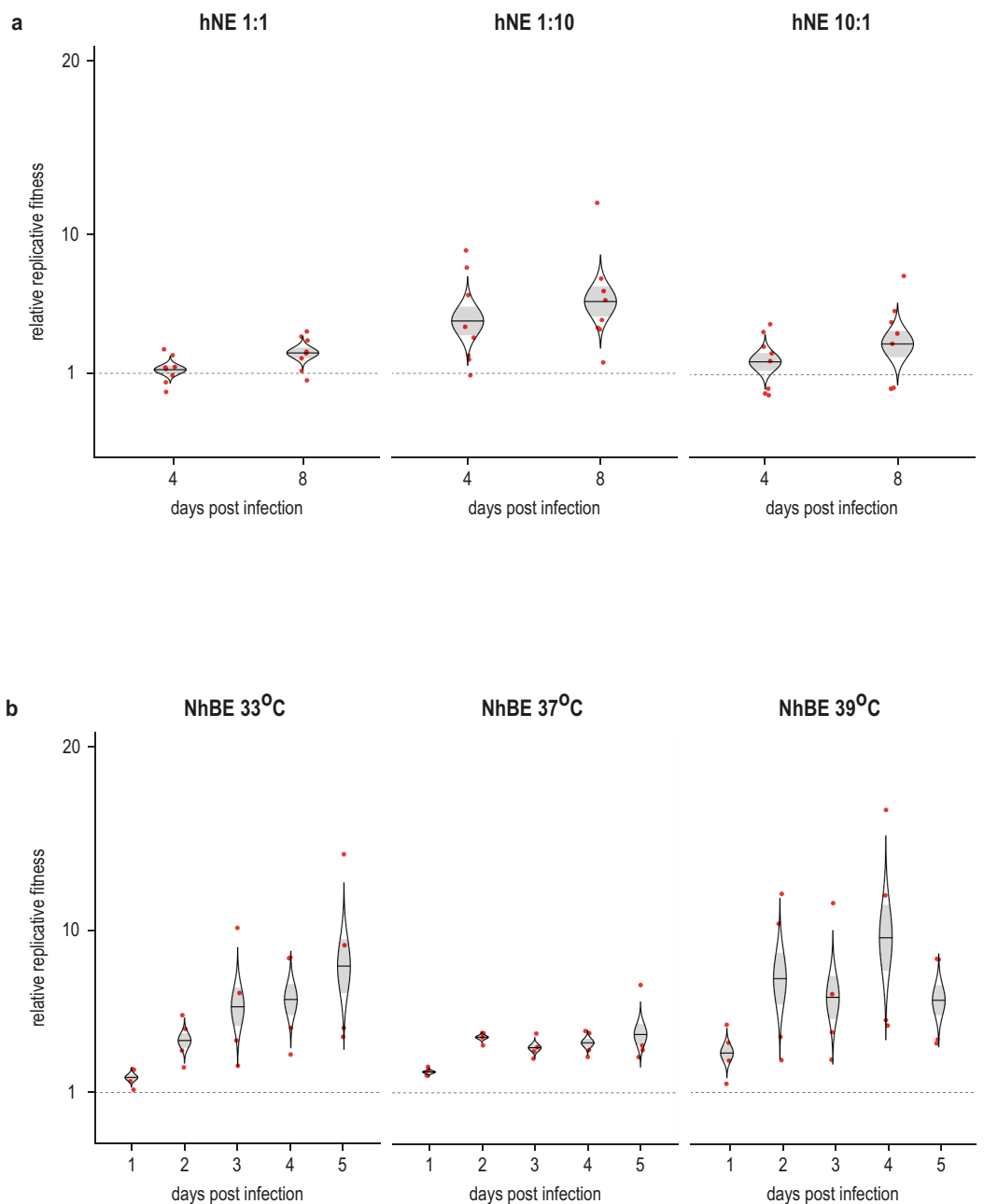

Extended Data Fig. 2 Relative replicative fitness of SARS-CoV-2 variants. a, b, Cat's eye plots illustrating the relative replicative fitness values of SARS-CoV-2 ${ }^{\mathrm{D} 614}$ over SARS-CoV-2 ${ }^{\mathrm{G} 614}$ in human nasal epithelial (a) and human
NBE (b) cells for the competition experiments shown in Fig. 1e, f. Each dot represents an individual data point, the centre line represents the mean and the shaded area represents the s.d. 


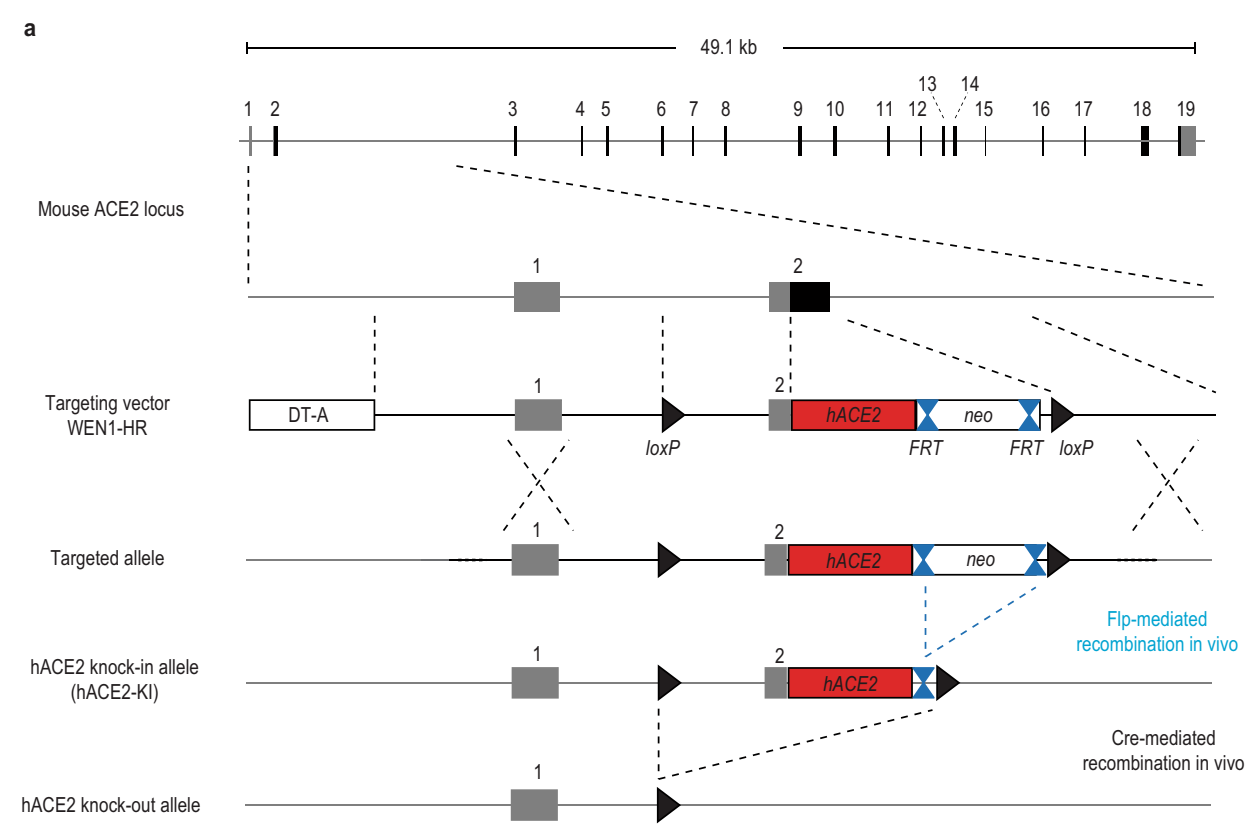

b

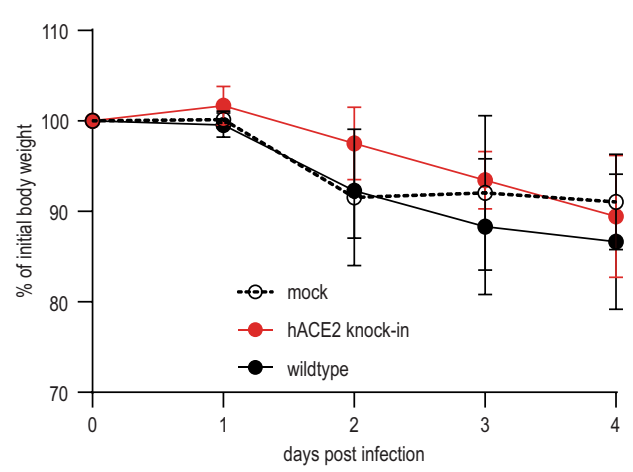

d

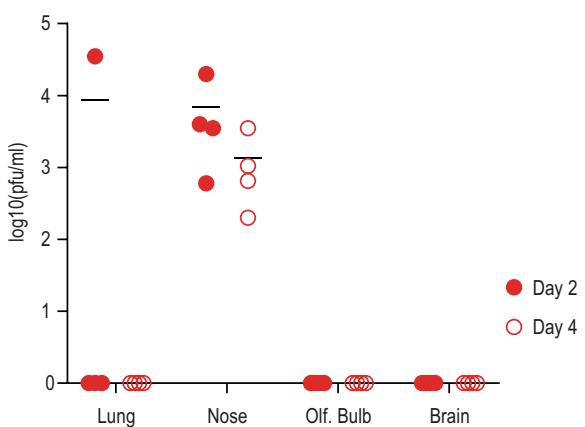

Extended Data Fig. 3 |See next page for caption.
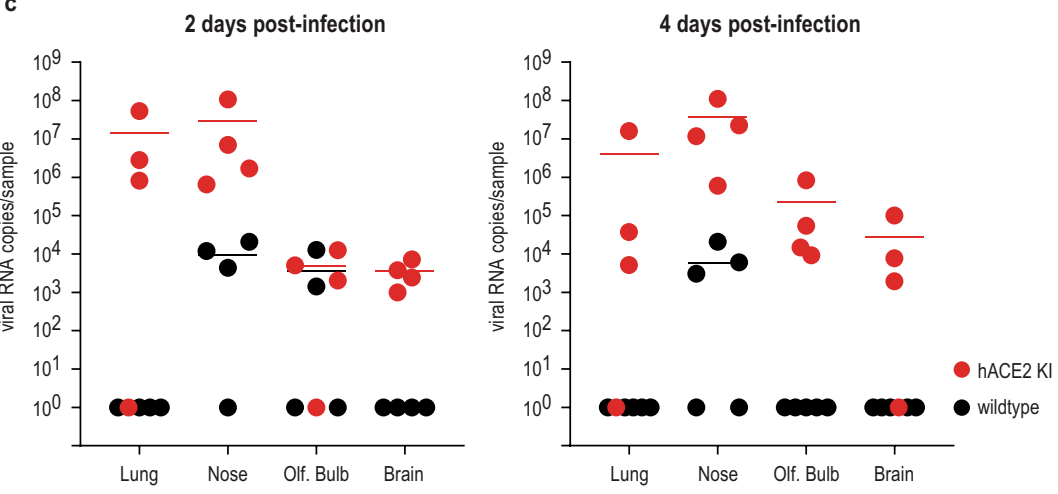

e

Oropharyngeal swab
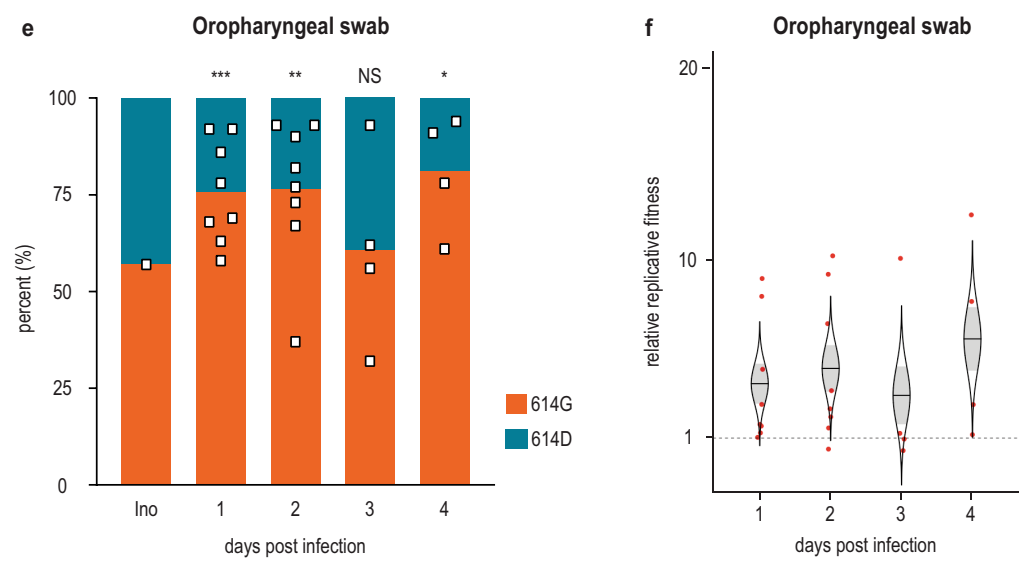

days post infection 


\section{Article}

Extended Data Fig. 3 | hACE2 knock-in mouse generation, and infection with SARS-CoV-2 ${ }^{\text {D614 }}$ and SARS-CoV-2 ${ }^{\text {G614 }}$. a, Design and generation of hACE2 knock-in mice. The human $A C E 2$ cDNA was inserted in-frame with the endogenous initiation codon of mouse Ace2 in exon 2, which was deleted. The human $A C E 2$ CDNA was flanked $5^{\prime}$ with a loxP site (black triangle) and $3^{\prime}$ with a FRT-neomycin-FR--loxP cassette. The targeting construct included a negative selection cassette (PGK-DTA) to improve selection of clones with homologous recombination. Chimeric male mice transmitting the targeted locus were crossed with Flp deleter female mice to generate the floxed human $A C E 2$ knockin allele. This allele can be used: (1) without further Cre-mediated recombination (as shown here) to study hACE2 knock-in mice, in which the human $A C E 2$ cDNA is expressed in place of mouse Ace2; (2) after crossing with a Cre-deleter mouse line to generate constitutive Ace2-knockout mice; and (3) after crossing with a tissue-specific Cre line. Ubiquitous and tissue-specific knockout mice can be crossed with conventional hACE2 transgenic mice to remove the endogenous mouse ACE2, which could confound pathogenesis studies (owing to heterodimerization of ACE2). b, Loss of body weight at indicated time points after infection of hACE2 knock-in mice $(n=8)$ and wildtype mice $(n=9)$, and for mock-infected wild-type mice sampled identically $(n=10) . \mathbf{c}, \mathbf{d}, \mathrm{RT}-\mathrm{qPCR}$ analysis $(\mathbf{c})$ and viral titres $(\mathbf{d})$ of tissue homogenates of inoculated hACE2 knock-in and wild-type mice at indicated time points. Olf., olfactory. e, Percentage of sequencing reads encoding S or S(D614G). Each square represents data for one individual mouse in a competition experiment. For each time point, a linear regression model was generated on the basis of the sequence read counts for $S$ and $S$ (D614G). $P$ values were calculated for the group (variant) coefficient. $P$ values (left to right): ${ }^{* * *} P=0.0009 ;{ }^{* *} P=0.0020$; NS, $P=0.7875$; and ${ }^{*} P=0.0180$. $f$, Cat's eye plot illustrating the relative replicative fitness values of SARS-CoV-2 ${ }^{\mathrm{D} 614}$ over SARS-CoV-2 ${ }^{\mathrm{G} 614}$ from oropharyngeal swabs of hACE2 knock-in mice in the competition experiment shown in Fig. 2. Ratios of SARS-CoV-2 $2^{\mathrm{G} 614}$ to SARS-CoV- ${ }^{\mathrm{D} 614}$ were measured after competition using the MinION sequencing platform at the time point indicated on the plot. Each dot represents one infected hACE2 knock-in mouse, the centre line represents the mean and the shaded area represents the s.d. 
a

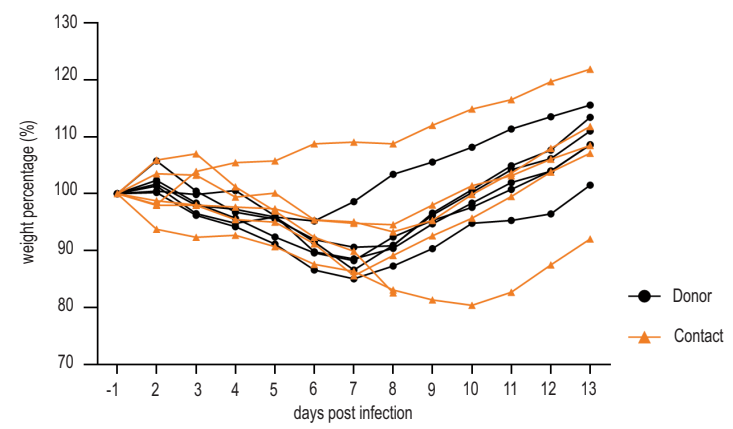

c

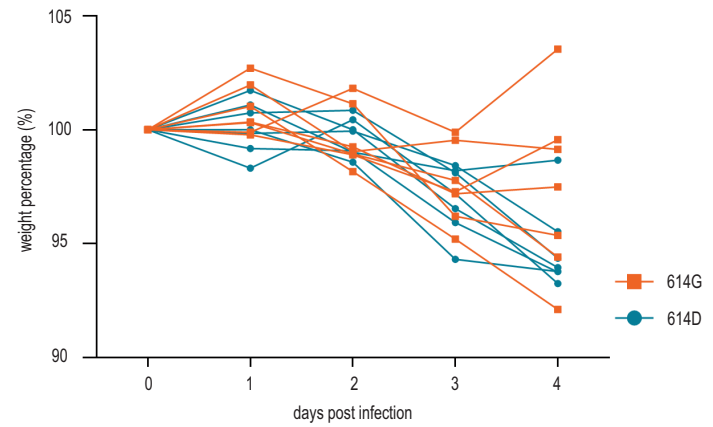

e
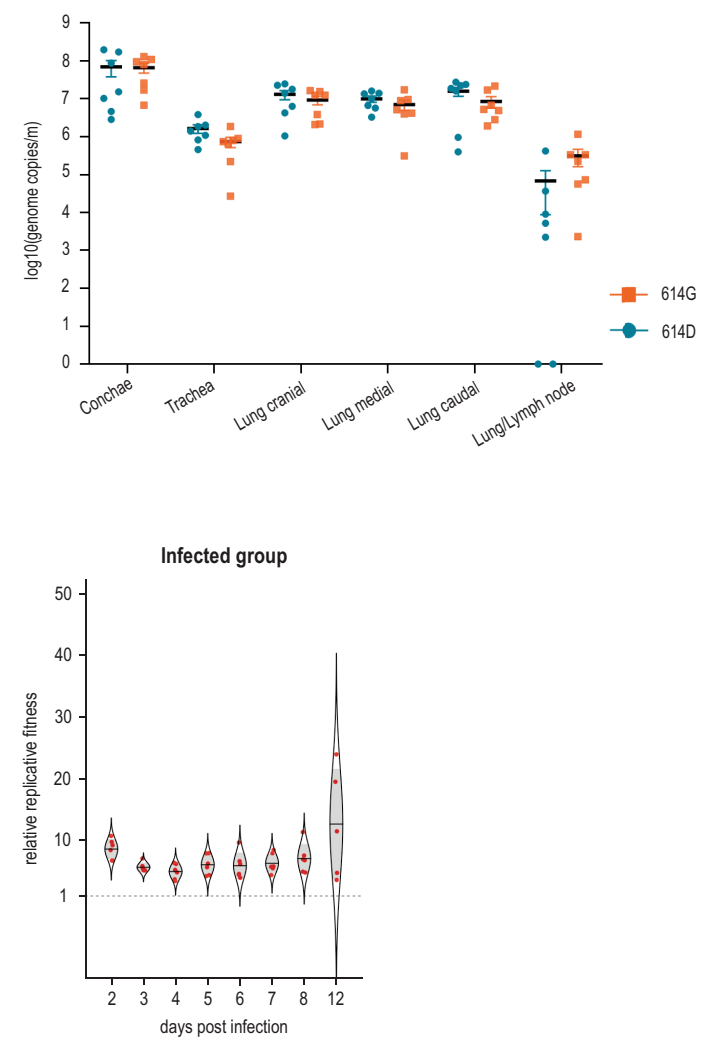

b

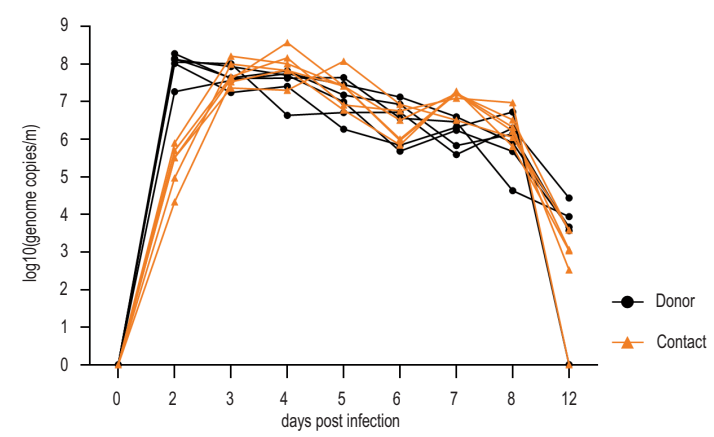

d

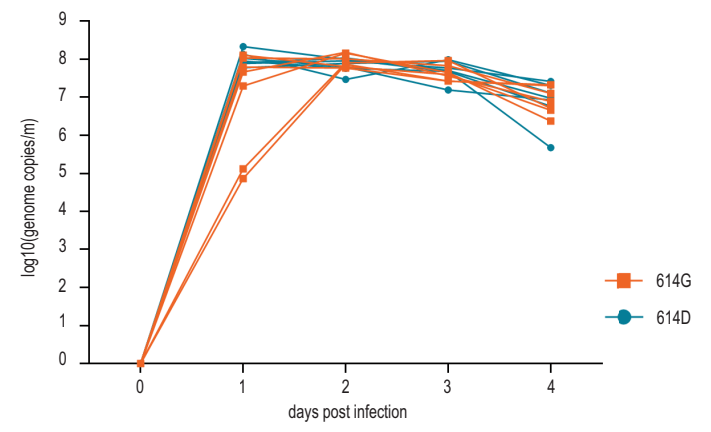

f

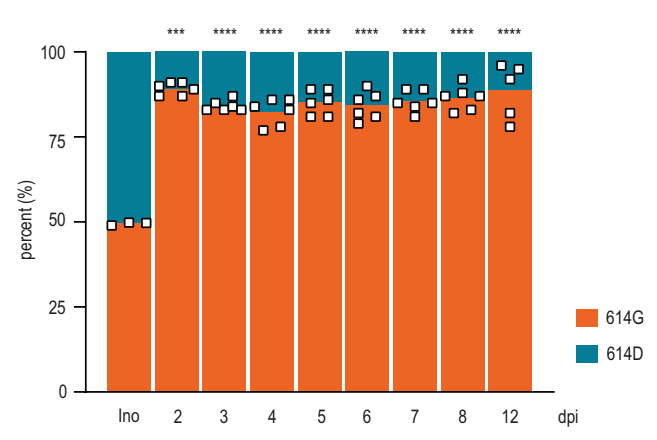

Extended Data Fig. 4 |See next page for caption. 


\section{Article}

Extended Data Fig. 4 | Virus replication in infected Syrian hamsters. a, Loss of body weight at indicated time points after infection of Syrian hamsters. Donor hamsters $(n=6)$ (black dots) were intranasally inoculated with SARSCoV-2 ${ }^{\mathrm{D} 614}$ and SARS-CoV- ${ }^{\mathrm{G} 614}$ at equal ratio $\left(1 \times 10^{4.77} \mathrm{TCID}_{50}\right.$ per hamster, as determined by back-titration of the original inoculum). Twenty-four $h$ after infection, naive hamsters $(n=6)$ (orange triangles) were housed in direct contact in a one-to-one experimental setup. b, RT-qPCR analysis of individual nasal washing samples obtained from donor and contact hamsters. $\mathbf{c}$, Loss of body weight at the indicated time points after infection of the hamsters. Syrian hamsters were inoculated with $10^{5.1} \mathrm{TCID}_{50}$ per hamster of SARS-CoV-2 ${ }^{\mathrm{D} 614}(n=7)$ (blue dots) or $10^{4.5} \mathrm{TCID}_{50}$ per hamster of SARS-CoV-2 ${ }^{\mathrm{G} 614}(n=7)$ (red triangles) via the intranasal route. Titres were determined by back titration of the original inoculation material. d, Viral genome copy numbers are shown as determined by RT-qPCR from individual nasal washing samples of the hamsters inoculated with single variant virus. e, RT-qPCR analysis of tissue homogenates of inoculated hamsters of the SARS-CoV-2 ${ }^{\mathrm{D} 614}$ group $(n=7)$ (blue dots) versus the SARS-CoV- $2^{\mathrm{G} 614}$ group $(n=7)$ (red triangles).f, Percentage of sequencing reads encoding S or S(D614G). Each square represents data for one individual hamster in the competition experiment. For each time point, a linear regression model was generated on the basis of the sequencing read counts for S and S(D614G). $P$ values were calculated for the group (variant) coefficient. ${ }^{* * *} P=0.0007 ;{ }^{* * * *} P<0.0001$. g, Cat's eye plot showing the relative replicative fitness values of SARS-CoV-2 ${ }^{\mathrm{G} 614}$ over SARS-CoV-2 ${ }^{\mathrm{D} 614}$ for infected hamsters from the competition experiment shown in Fig. 3. Ratios of SARS-CoV- $2^{\mathrm{G} 614}$ to SARS-CoV-2 ${ }^{\mathrm{D} 614}$ were measured after competition using the MinION sequencing platform at the time points indicated on the plot. Each dot represents one infected hamster $(n=6)$, the centre line represents the mean and the shaded area represents the s.d. 


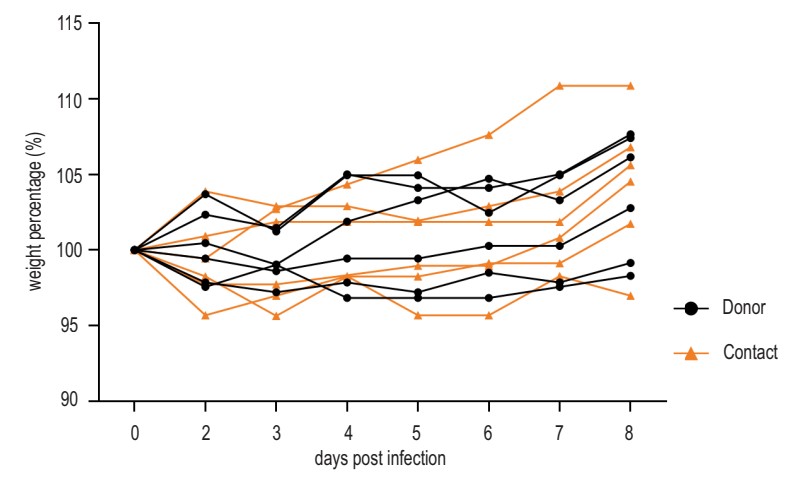

c

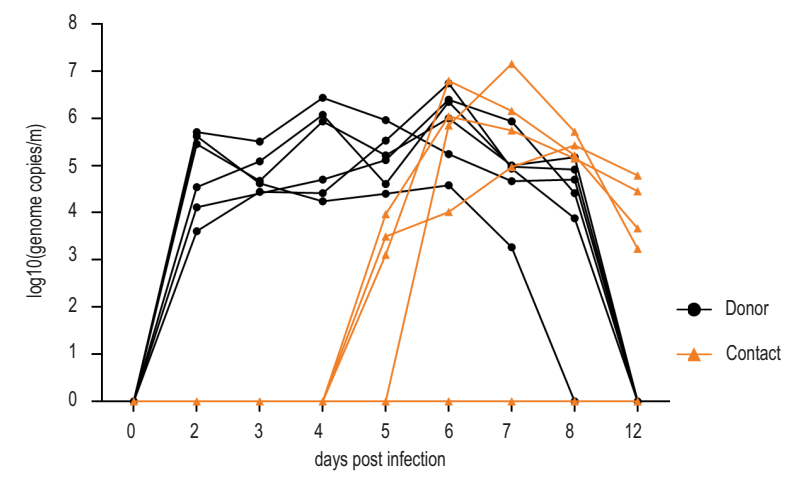

d

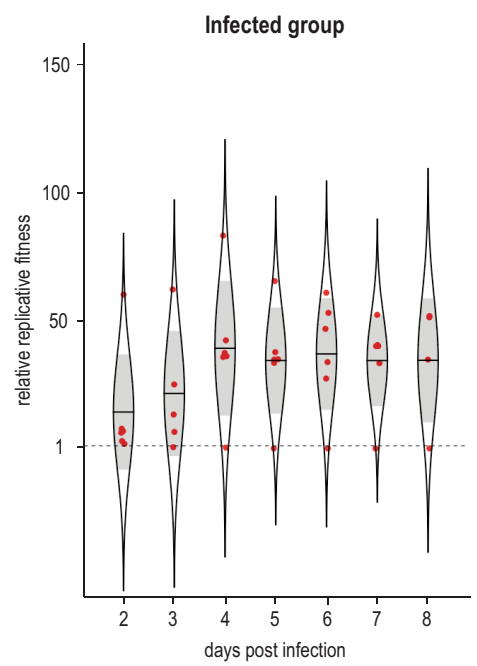

Extended Data Fig. 5 | Twin inoculation of donor ferrets with equal ratios of SARS-CoV-2 ${ }^{\text {D614 }}$ and SARS-CoV-2 ${ }^{\mathbf{6} 14}$. Donor ferrets (black dots) $(n=6)$ were intranasally inoculated with $10^{5.4} \mathrm{TCID}_{50}$ per ferret, as determined by back titration of an inoculum comprising equal ratios of SARS-CoV-2 ${ }^{\mathrm{D} 614}$ and SARS-CoV-2 ${ }^{\mathrm{G} 614}$. Twenty-four hours after inoculation, one contact ferret (orange triangle) $(n=6)$ was commingled with one donor ferret, creating six donor-contact ferret pairs. a, Individual body weight of ferrets at the indicated days relative to the day of inoculation is plotted. $\mathbf{b}$, Genome copy numbers for inoculated donor and contact ferrets. Individual nasal washing samples of the indicated days were analysed by RT-qPCR nCoV_IP4, and absolute numbers were calculated using a set of standard RNA. All donor ferrets (black dots) tested positive for viral RNA, starting from day 2 after inoculation $(n=6)$. Four out of six contact ferrets (orange triangles) tested positive for viral RNA, starting from day 4 (corresponding to day 3 after contact). Two of the six

contact ferrets never tested positive for viral RNA throughout the study. c, Percentage of sequencing reads encoding S or S(D614G). Each square represents data for one individual ferret in the competition experiment. For each time point, a linear regression model was generated on the basis of the sequence read counts for $S$ and S(D614G). $P$ values were calculated for the group (variant) coefficient. $P$ values (left to right): ${ }^{* * *} P=0.0001 ; * * P=0.0090$; ${ }^{*} P=0.0030 ;{ }^{*} P=0.0352 ;{ }^{*} P=0.0393 ;{ }^{*} P=0.0411$; and NS,$P=0.2883$. d, Cat's eye plot illustrating the relative replicative fitness values of SARS-CoV-2 ${ }^{\mathrm{G} 614}$ over SARS-CoV-2 ${ }^{\mathrm{D} 614}$ in infected ferrets from the competition experiment shown in Fig. 4. Ratios of SARS-CoV-2 ${ }^{\mathrm{G} 614}$ to SARS-CoV-2 ${ }^{\mathrm{D} 614}$ were measured after competition using the MinION sequencing platform at the time points indicated on the plot. Each dot represents one infected ferret $(n=6)$, the centre line represents the mean and the shaded area represents the s.d. 


\section{Reporting Summary}

Nature Research wishes to improve the reproducibility of the work that we publish. This form provides structure for consistency and transparency in reporting. For further information on Nature Research policies, see Authors \& Referees and the Editorial Policy Checklist.

\section{Statistics}

For all statistical analyses, confirm that the following items are present in the figure legend, table legend, main text, or Methods section.

n/a Confirmed

$\square \bigotimes$ The exact sample size $(n)$ for each experimental group/condition, given as a discrete number and unit of measurement

$\square \bigotimes$ A statement on whether measurements were taken from distinct samples or whether the same sample was measured repeatedly

The statistical test(s) used AND whether they are one- or two-sided

$\triangle$ Only common tests should be described solely by name; describe more complex techniques in the Methods section.

$\triangle \square$ A description of all covariates tested

$\square$ A description of any assumptions or corrections, such as tests of normality and adjustment for multiple comparisons

$\square$ A full description of the statistical parameters including central tendency (e.g. means) or other basic estimates (e.g. regression coefficient)

$\triangle$ AND variation (e.g. standard deviation) or associated estimates of uncertainty (e.g. confidence intervals)

$\searrow$ For null hypothesis testing, the test statistic (e.g. $F, t, r$ ) with confidence intervals, effect sizes, degrees of freedom and $P$ value noted

Give P values as exact values whenever suitable.

Х $\square$ For Bayesian analysis, information on the choice of priors and Markov chain Monte Carlo settings

Х $\square$ For hierarchical and complex designs, identification of the appropriate level for tests and full reporting of outcomes

$\bigotimes \square$ Estimates of effect sizes (e.g. Cohen's $d$, Pearson's $r$ ), indicating how they were calculated

Our web collection on statistics for biologists contains articles on many of the points above.

\section{Software and code}

Policy information about availability of computer code

Data collection Data acquisition for Minion sequencing was done with MinKNOW version 20.06.17 and basecalling software Guppy version 4.0.11

Data analysis $\quad$ seqeunce analysis: Geneious Prime ${ }^{\circledR} 2019.2 .3$

virus kinetics analyses: GraphPad Prism version 8.3.0 for Windows

figures: Adobe Illustrator CS6; Catseyes version 0.2.5; R version 4.0.2

NGS (RNAseq): TrimGalore software (version 0.6.5), SAMtools (version 1.10); Bowtie2 version 2.3.5

Minion sequencing: MinKnow version 20.06.17; Guppy version 4.0.11

digital PCR: QuantaSoft Analysis Pro software (version 1.0.596)

FACS: Flowjo_v10.6.1.

For manuscripts utilizing custom algorithms or software that are central to the research but not yet described in published literature, software must be made available to editors/reviewers. We strongly encourage code deposition in a community repository (e.g. GitHub). See the Nature Research guidelines for submitting code \& software for further information.

\section{Data}

Policy information about availability of data

All manuscripts must include a data availability statement. This statement should provide the following information, where applicable:

- Accession codes, unique identifiers, or web links for publicly available datasets

- A list of figures that have associated raw data

- A description of any restrictions on data availability 


\section{Field-specific reporting}

Please select the one below that is the best fit for your research. If you are not sure, read the appropriate sections before making your selection. $\bigotimes$ Life sciences $\quad \square$ Behavioural \& social sciences $\quad \square$ Ecological, evolutionary \& environmental sciences

For a reference copy of the document with all sections, see nature.com/documents/nr-reporting-summary-flat.pdf

\section{Life sciences study design}

All studies must disclose on these points even when the disclosure is negative.

Sample size No sample size calculations were performed. Sample sizes were based on standards in the field, typically 3 independent biological replicates, with each replicate assayed in technical duplicate or triplicate. Experiments involving hNE and NhBE primary airway cultures were done in technical replicates since these cells are purchased and are delivered with a quality guarntee for the delivery data. Therefore only technical replicates were performed.

Data exclusions no data was excluded

Replication all attempts at replication were successful; experiments were performed according to best practices and as described in the methods.

Randomization randomization was not applied since cloning procedures, virus infection/titrations, and inhibitor/neutralization experiments did not require randomization.

Blinding blinding was done for seqeuncing analyses for the determination of the ratio of genotypes 614D vs 614G. For all other experiments blinding was not done.

\section{Reporting for specific materials, systems and methods}

We require information from authors about some types of materials, experimental systems and methods used in many studies. Here, indicate whether each material, system or method listed is relevant to your study. If you are not sure if a list item applies to your research, read the appropriate section before selecting a response.

\begin{tabular}{|c|c|c|c|}
\hline \multicolumn{2}{|r|}{ Materials \& experimental systems } & \multicolumn{2}{|c|}{ Methods } \\
\hline $\mathrm{n} / \mathrm{a}$ & Involved in the study & $\mathrm{n} / \mathrm{a}$ & Involved in the study \\
\hline & \ Antibodies & Х & $\square$ ChIP-seq \\
\hline L & ¿ Eukaryotic cell lines & & $\bigotimes$ Flow cytometry \\
\hline$\bigotimes$ & Palaeontology & Х & MRI-based neuroimaging \\
\hline 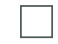 & \ Animals and other organisms & & \\
\hline$\bigotimes$ & $\square$ Human research participants & & \\
\hline Х & Clinical data & & \\
\hline
\end{tabular}

\section{Antibodies}

Antibodies used

Goat anti-Human IgG (H+L) Cross-Adsorbed Secondary Antibody, Alexa Fluor 633, 1:300 dilution (ThermoFisher Cat \# A-21091 for Fc tag)

6x-His Tag Monoclonal Antibody (HIS.H8), Alexa Fluor 647, 1:300 dilution (ThermoFisher Cat \# MA1-21315-647 for polyhistidinetag)

Validation

No primary antibodies were used in this study. The only things that are "similar to" primary antibodies are His-tagged or Fctagged S1 proteins for hACE2 detection in FACS, which were validated by BLI assay and ELISA assay so confirm they can bind to hACE2.

\section{Eukaryotic cell lines}

Policy information about cell lines

Cell line source(s)

VeroE6 (obtained from Marcel Müller, Charité, Berlin)

BHK-SARS-N (prepared and published in: van den Worm, S. H. et al. Reverse genetics of SARS-related coronavirus using vaccinia virus-based recombination. PLoS One 7, e32857, doi:PONE-D-11-21011)

BHK-hACE2 (perpared in this study) 


\section{Authentication}

Mycoplasma contamination

Commonly misidentified lines (See ICLAC register)
Profiling of cell line was done using highly-polymorphic short tandem repeat loci (STRs). Fragment analysis was done on an ABI3730xI (Life Technologies) and the resulting data were analyzed with GeneMarker software (Softgenetics).

all cell lines in our laboratories are routinely screened for micoplasma contamination and were tested negative.

none

\section{Animals and other organisms}

Policy information about studies involving animals; ARRIVE guidelines recommended for reporting animal research

Laboratory animals

Mice. 10-12 weeks old. Female heterozygous Ace2<tm1(ACE2)Dwnt>/J mice were used. They were housed at $22^{\circ} \mathrm{C}$ ambient temperature and $50 \%$ humidity. Detailed experimentation protocols and are fully disclosed according the ARRIVE guidlines in the methods' section of the manuscript.

Ferrets. 4-9 months old ferrets (Mustela putorius furo, 4 female and 8 male) were infected intranasally with the indicated viruses. They were housed at $22^{\circ} \mathrm{C}$ ambient temperature and $50 \%$ humidity.

Hamsters. 8 weeks old Syrian hamsters (Mesocricretus auratus, 26 male) were infected intranasally with the indicated viruses. They were housed at $22^{\circ} \mathrm{C}$ ambient temperature and $50 \%$ humidity.

Wild animals

no wild animals were used in the study.

Field-collected samples

Ethics oversight

\section{no field collected samples were used in the study.}

Mice. The hACE-2 knock-in mice (B6.129S2(Cg)-Ace2<tm1(ACE2)Dwnt>/J) were originally generated at the Wadsworth Center, New York State Department of Health IACUC protocol \# 09-405 (Wentworth, PI). Mouse experimentation was conducted at the Institute of Virology and Immunology, Mittelhäusern, Switzerland in compliance with the Swiss Animal Welfare legislation and animal studies were reviewed and approved by the commission for animal experiments of the canton of Bern, Switzerland under license BE-43/20.

Ferrets and hamsters. All ferret and hamster experiments were evaluated by the responsible ethics committee of the State Office of Agriculture, Food Safety, and Fishery in Mecklenburg-Western Pomerania, Germany (LALLF M-V), and gained governmental approval under registration number LVL MV TSD/7221.3-1- 041/20.

Note that full information on the approval of the study protocol must also be provided in the manuscript.

\section{Flow Cytometry}

\section{Plots}

Confirm that:

$\bigotimes$ The axis labels state the marker and fluorochrome used (e.g. CD4-FITC).

ХThe axis scales are clearly visible. Include numbers along axes only for bottom left plot of group (a 'group' is an analysis of identical markers).

$\bigotimes$ All plots are contour plots with outliers or pseudocolor plots.

\A numerical value for number of cells or percentage (with statistics) is provided.

\section{Methodology}

Sample preparation

Instrument

BHK cells expressing exogenous hACE2 were pelleted and resuspended in reaction buffer (PBS pH7.4, 0.02\% tween20, BSA 4\%) at a concentration of $5 \times 106 \mathrm{cell} / \mathrm{s} / \mathrm{ml}$. $100 \mathrm{ul} /$ well of the cells were aliquoted into a round-bottom 96-well plate and incubated on ice for at least $5 \mathrm{~min}$. S1 proteins were diluted in reaction buffer on ice. 50ul of S1 diluents were added into corresponding wells of cells and incubated on ice for 20 min with shaking. After incubation, cells were washed in 200ul washing solution (PBS pH7.4, $0.02 \%$ tween 20) once and then 100ul of 1:300 diluted secondary antibody (ThermoFisher Cat \# A-21091 for Fc tag and ThermoFisher Cat \# MA1-21315-647 for polyhistidine-tag) was added into each well of cells, mixed, and incubated on ice with shaking for $15 \mathrm{~min}$. After washing twice, cells were resuspended in 200ul PBST and analyzed using the BD FACSCanto II Flow Cytometer. Data was processed with Flowjo_v10.6.1.

\section{Software}

\section{BD FACSCanto II Flow Cytometer}

Flowjo_v10.6.1.

Cell population abundance

Gating strategy

\section{BHK cells expressing hACE2 were used as the only cell population}

BHK cells expressing hACE2 were used without S1Fc or S1-His and mean fluorescence intesity was measured in comparison with BHK cells expressing hACE2 incubated with indicated concentrations of S1-Fc or S1-His. Shifts in mean fluorescence intensity were recoreded and displayed in Fig $1 \mathrm{~b}$ and Extended Data Figure $1 \mathrm{~b}$.

$\bigotimes$ Tick this box to confirm that a figure exemplifying the gating strategy is provided in the Supplementary Information. 